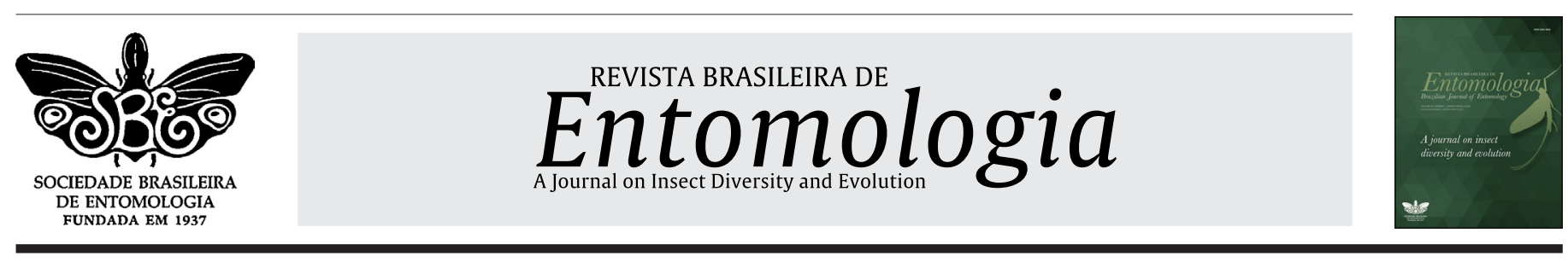

\title{
New records and expansion of the geographic distribution of gall inducers of the family Cecidomyiidae (Diptera) associated to Andira Lam. (Fabaceae) species in Brazil
}

\author{
Eduardo Alves Martins Silva ${ }^{1}\left(\mathbb{C}\right.$, Maria Virginia Urso-Guimarães ${ }^{*}{ }^{*}$ () \\ ${ }^{1}$ Universidade Federal de São Carlos, Departamento de Biologia, Laboratório de Sistemática de Diptera, Sorocaba, SP, Brasil.
}

\section{A R T I C L E I N F O}

Article history:

Received 05 July 2021

Accepted 29 September 2021

Available online 27 October 2021

Associate Editor: Marcia Couri

\section{Keywords:}

Andira fraxinifolia

Andira humilis

Andira nítida

Andira vermífuga

Insect-plant interaction

\begin{abstract}
A B S T R A C T
Galls are structures of vegetal tissue modified by several biological agents, mainly dipterans of the Cecidomyiidae family. The galls are indicators of the gall inducers' presence, because of the species-specific interaction between the gall inducer and its host plant species. The aim of this study was to expand the geographical distribution of cecidomyiids - usually known only to the type-locality - in species of Andira Lam. (Fabaceae) distributed in Brazil, through the presence of their gall morphotypes in host plants. We searched for records of gall morphotypes in Andira species in all inventories of insect galls in Brazilian biomes and in virtual herbaria in Brazilian and abroad institutions. We found six species of Andira with forty-nine registers of 20 morphotypes of galls induced by cecidomyiids. They were from 35 localities; of those, 15 Municipalities, five States and the Federal District are new points of occurrence. We found three new registers for the fusiform gall in $A$. fraxinifolia, four registers for the fusiform gall in $A$. humilis, two new registers for the lenticular gall in $A$. nitida, and six new registers for three morphotypes in $A$. vermifuga. No new records were found for the morphotypes of $A$. cujabensis and $A$. surinamensis. These results indicate that digital tools, combined with well-illustrated inventory data, are capable to expand knowledge about the distribution of cecidomyiids through the recognition of their specific marker, the morphotypes.
\end{abstract}

\section{Introduction}

Galls are the result of an abnormal growth process of cells, tissues or vegetal organs in response to the activity of biological agents as viruses, bacteria, nematodes, and insects (Shorthouse and Rohfritsch, 1992; Shorthouse et al., 2005; Carneiro et al., 2009).

The gall-inducer insects have a highly specific interaction with host plants (Isaias et al., 2014), generating unique morphotypes that allow identifying it through specific characteristics, such as organ of occurrence, shape, color, size, indumentum number of internal locus (Isaias et al., 2014). In the case of cecidomyiids, $92.4 \%$ of the species are monophagous (Carneiro et al., 2009), which allow us to assume the identification of the gall inducer through the presence of its morphotype in the specific plant species (Skuhravá et al., 2014). The external morphology of the gall is a reliable marker of this association (EspíritoSanto and Fernandes, 2007).

Arriola et al. (2016) and Lima and Calado (2020) showed the overlap in the distribution of the host plant and the gall inducer, which allows us to infer that the distribution of the gall inducer cecidomyiids species are circumscribed to the host plant distribution. The geographical occurrence of gall inducers may not be uniform due to several factors, including variation in natural plant composition and geographical

\footnotetext{
${ }^{*}$ Corresponding author.

E-mail: mvirginiaurso@gmail.com (M.V. Urso-Guimarães).
}

position, especially in the North-South axis, with latitude-related variations (Skuhravá and Skuhravý, 2009).

The genus Andira Lam. belongs to Fabaceae, one of the richest families of the world with 3,033 species and in Brazil, with 1,578 species (Pennington, 2003). Andira has 29 species in the tropical regions of America, 20 of them confirmed in all Brazilian regions. Andira species occur in Amazonia, Caatinga, Cerrado, Atlantic Forest phytogeographic domains. They are very diverse and occupy various habitats such as Caatinga, Campinarana, Cerrado, Riparian Forest, Amazon flood forests, Dry Seasonal Forests, Ombrophile Forest, Restinga, as well as anthropized areas. They have habits such as shrubs and trees that grow in different soil fertility conditions, from nutrient-rich soils to poor rocky soils (Ramos et al., 2020).

Currently, four species of cecidomyiids are known inducing galls in Andira: Lopesia indaiensis Maia and Oliveira, 2018, gall inducer in A. fraxinifolia Benth. recorded in Minas Gerais (Dores do Indaiá) in Cerrado and in Pernambuco (Tamandaré), Rio de Janeiro (Mangaratiba), and São Paulo (Bertioga) in Atlantic forest (Maia, 2021); Lopesia andirae Garcia et al., 2017 gall inducer in A. humilis Mart. ex Benth. occurring in Cerrado areas in Mato Grosso (Chapada dos Guimarães), Bahia (Barreiras), and São Paulo (Luiz Antônio) (Garcia et al., 2017); $L$. chapadensis Garcia and Urso-Guimarães, 2018 inductor in $A$. vermifuga (Mart.) Benth. occurring in a Cerrado area of Mato Grosso (Chapada 
dos Guimarães) (Garcia and Urso-Guimarães, 2018), and Andirodiplosis bahiensis Tavares, 1920 inductor of "sinuous" leaf galls in an unidentified species of Andira in Bahia (Tavares, 1920).

Considering: (i) the high specificity of gall inducer and host plant species interaction, (ii) that the morphotype is a reliable marker of the interaction; and (iii) that the distribution of the gall inducer is circumscribed to the distribution of the host plant, in this work we aimed to verify matching between distribution of the cecidomyiids species and the Andira species in Brazil and to register the new geographical records using literature and virtual herbaria data.

\section{Material and methods}

The review of literature and exsiccates in virtual herbaria are important tools to increase the scope of search for distribution data of host plants and, consequently, to the associated gall inducer as showed by Arriola et al. (2016). In our study, we used the Web of Science, Scielo, Periódicos Capes, and Google Scholar as tools to search using the words "inventários de galhas", “Andira”, "galhas Andira” "Cecidomyiidae Andira” in English and in Portuguese by December 2020 with no limitation on time period. All registers found were included. For searching gall morphotypes in Andira species we used the virtual herbaria in Brazilian and abroad institutions through the tools INCT Herbário Virtual da Flora e dos Fungos (http://inct.florabrasil.net/category/reflora/) and SpeciesLink (http://splink.cria.org.br/tools) using the word "Andira".

The herbaria where the exsiccates with Andira galls were found are as follows: Herbário da Embrapa CENARGEN (CEN), Herbário do Jardim Botânico do Rio de Janeiro (JBJR), Royal Botanic Gardens, Kew
(K), Herbário do Museu de Biologia Prof. Mello Leitão (MBML), Muséum National d'Histoire Naturelle (MNHN), New York Botanical Garden Herbarium (NY), Herbário da Universidade Federal de Campinas (UEC), and Herbário Rondoniense João Geraldo Kuhlmann, da Universidade Federal de Rondônia (RON). We checked the world catalogue of Cecidomyiidae (Gagné and Jaschhof, 2021) for the species of cecidomyiid associated to the Andira species. The maps were built with Qgis program (QGIS Development Team, 2020) using a shapefile from Brazil (IBGE, 2019) and the coordinates found in the inventories and data on digital collection of herbaria. The morphotype nomenclature was updated based on the proposal of Araújo et al. (2021).

\section{Results}

The searches carried out in virtual herbaria including 241 exsiccates of $A$. cujabensis, 1,105 of $A$. fraxinifolia, 502 of $A$. humilis, 291 of $A$. nitida, 185 of $A$. surinamensis, and 274 of $A$. vermifuga. Among them, we found 49 records of 20 morphotypes of galls in the six species of Andira. The data of morphotypes, cecidomyiid species associated, and the occurrence points are summarized in Table 1.

All the six species of Andira discussed in this paper are native and endemic from Brazil, except for $A$. surinamensis, also distributed in Trinidad and Tobago, Colombia, Venezuela, Guiana, French Guiana, Suriname, Ecuador, Peru, and Bolivia, and $A$. vermifuga distributed in Peru and Bolivia.

Andira cujabensis is widely distributed in Brazil, in Amazonia and Cerrado phytogeographic domains (Ramos et al., 2020) (Fig. 1). Despite this, only one inventory of insect galls registered the four morphotypes

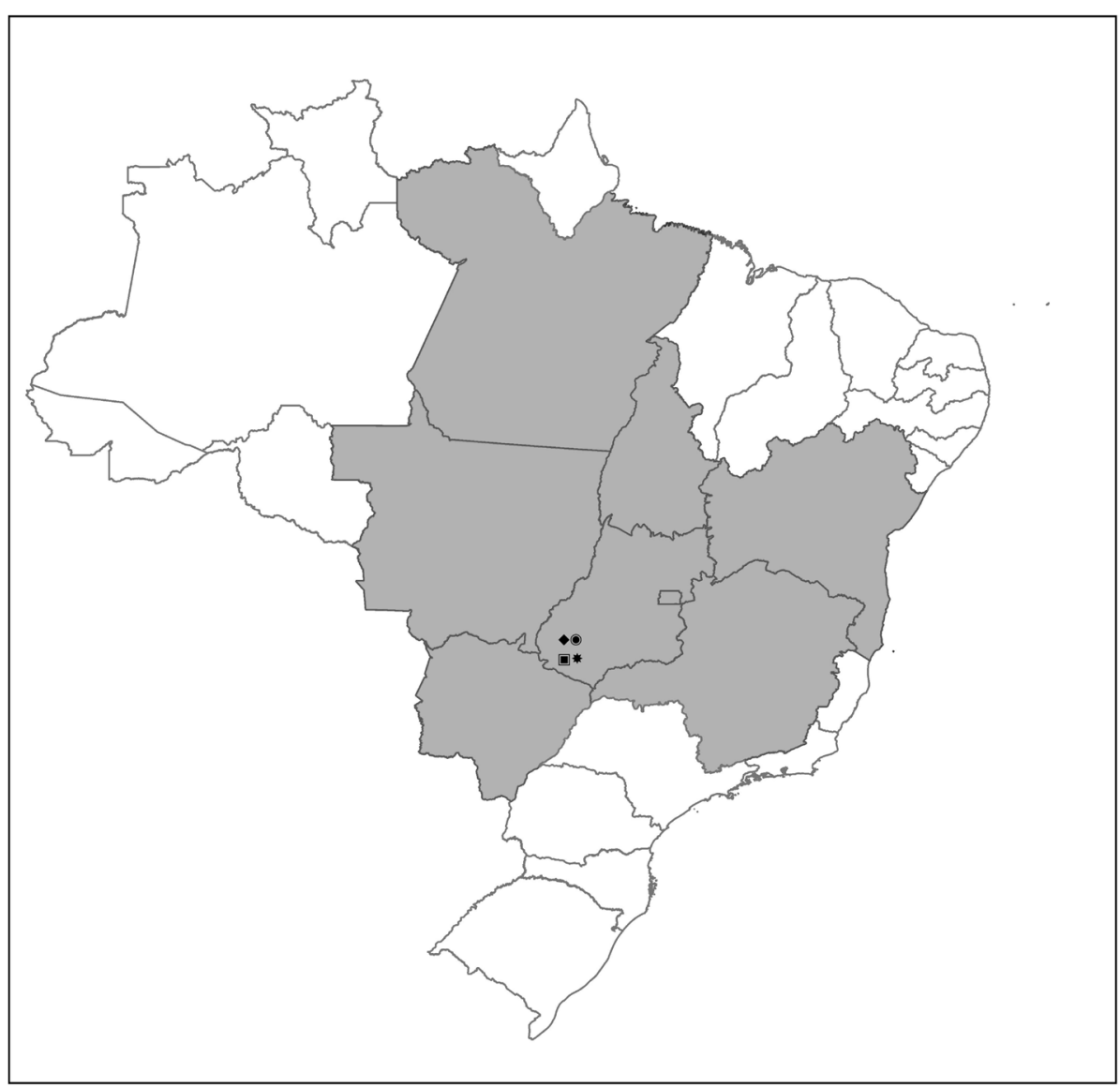

Occurrence of Andira cujabensis Benth. in Brazilian states

No occurrence Confirmed occurrence

\section{Gall morphotypes in Andira cujabensis Benth. in Brazilian state}

\begin{tabular}{|l|c|}
\hline \multicolumn{2}{|c|}{ Gall morphotypes } \\
\hline Stem globoid & \\
\hline Leaf lenticular & 0 \\
\hline Leaf conical & $\square$ \\
\hline Leaf fusiform & \\
\hline
\end{tabular}

Figure 1 Distribution of Andira cujabensis Benth. in Brazilian states and the point of occurrence of the gall morphotypes induced in the species. 
Table 1

Previous and new records of galls on Andira species (Fabaceae) in Brazil.

\begin{tabular}{|c|c|c|c|c|c|c|c|c|}
\hline Host plant & $\begin{array}{c}\text { Organ/ } \\
\text { Morphotype }\end{array}$ & $\begin{array}{l}\text { Number of gall } \\
\text { morphotype } \\
\text { (GM) }\end{array}$ & Gall inducer & $\begin{array}{l}\text { Municipality/ } \\
\text { State }\end{array}$ & $\begin{array}{c}\text { Phytogeographic } \\
\text { domain }\end{array}$ & Coordinates & Reference & Figure \\
\hline \multirow[t]{4}{*}{$\begin{array}{l}\text { Andira cujabensis } \\
\text { Benth. }\end{array}$} & Leaf/Conical & GM1 & $\begin{array}{l}\text { Undetermined sp } \\
1 \text { (Cecidomyiidae) }\end{array}$ & Mineiros/GO & Cerrado & $\begin{array}{c}17^{\circ} 49^{\prime}-18^{\circ} 28^{\prime} \mathrm{S} / 52^{\circ} 39^{\prime}- \\
53^{\circ} 10^{\prime} \mathrm{W}\end{array}$ & $\begin{array}{l}\text { Araújo et al. } \\
\text { (2014) }\end{array}$ & Unavailable \\
\hline & Leaf/Fusiform & GM2 & $\begin{array}{l}\text { Undetermined sp } \\
2 \text { (Cecidomyiidae) }\end{array}$ & Mineiros/GO & Cerrado & $\begin{array}{c}17^{\circ} 49^{\prime}-18^{\circ} 28^{\prime} \mathrm{S} / 52^{\circ} 39^{\prime}- \\
53^{\circ} 10^{\prime} \mathrm{W}\end{array}$ & $\begin{array}{l}\text { Araújo et al. } \\
\qquad(2014)\end{array}$ & Unavailable \\
\hline & Leaf/Lenticular & GM3 & $\begin{array}{l}\text { Undetermined sp } \\
3 \text { (Cecidomyiidae) }\end{array}$ & Mineiros/GO & Cerrado & $\begin{array}{c}17^{\circ} 49^{\prime}-18^{\circ} 28^{\prime} \mathrm{S} / 52^{\circ} 39^{\prime}- \\
53^{\circ} 10^{\prime} \mathrm{W}\end{array}$ & $\begin{array}{l}\text { Araújo et al. } \\
\qquad(2014)\end{array}$ & Unavailable \\
\hline & Stem/Globoid & GM4 & $\begin{array}{l}\text { Undetermined sp } \\
4 \text { (Cecidomyiidae) }\end{array}$ & Mineiros/GO & Cerrado & $\begin{array}{c}17^{\circ} 49^{\prime}-18^{\circ} 28^{\prime} \mathrm{S} / 52^{\circ} 39^{\prime}- \\
53^{\circ} 10^{\prime} \mathrm{W}\end{array}$ & $\begin{array}{l}\text { Araújo et al. } \\
\qquad(2014)\end{array}$ & Unavailable \\
\hline \multirow[t]{19}{*}{$\begin{array}{c}\text { Andira } \\
\text { fraxinifolia Benth. }\end{array}$} & Leaf/Fusiform & GM5 & $\begin{array}{c}\text { Lopesia } \\
\text { indaiensis } \\
\text { (Cecidomyiidae) }\end{array}$ & $\begin{array}{c}\text { Dores do Indaiá/ } \\
\text { MG }\end{array}$ & Cerrado & $\begin{array}{c}19^{\circ} 30^{\prime} 18.99^{\prime \prime} \mathrm{S} / 45^{\circ} 41^{\prime} 00.88^{\prime \prime} \\
\mathrm{W}\end{array}$ & $\begin{array}{l}\text { Maia and de } \\
\text { Oliveira (2018) }\end{array}$ & Fig. $3 \mathrm{~A}$ \\
\hline & Leaf/Fusiform & GM5 & $\begin{array}{c}\text { Lopesia } \\
\text { indaiensis } \\
\text { (Cecidomyiidae) }\end{array}$ & Bertioga/SP & Atlantic Forest & $23^{\circ} 51^{\prime} 16^{\prime \prime} \mathrm{S} / 46^{\circ} 08^{\prime} 19^{\prime \prime} \mathrm{W}$ & $\begin{array}{l}\text { Maia et al. } \\
\text { (2008) }\end{array}$ & Fig. 3B \\
\hline & Leaf/Fusiform & GM5 & $\begin{array}{c}\text { Lopesia } \\
\text { indaiensis } \\
\text { (Cecidomyiidae) }\end{array}$ & Mangaratiba/RJ & Atlantic Forest & $\begin{array}{l}\text { 233'57.34"S/4359'31.01"W, } \\
23^{\circ} 3^{\prime} 27.08^{\prime \prime} \mathrm{S} / 43^{\circ} 59^{\prime} 28.63^{\prime \prime W}\end{array}$ & $\begin{array}{l}\text { Rodrigues et al. } \\
\qquad(2014)\end{array}$ & Fig. $3 \mathrm{C}$ \\
\hline & Leaf/Fusiform & GM5 & $\begin{array}{c}\text { Lopesia } \\
\text { indaiensis } \\
\text { (Cecidomyiidae) }\end{array}$ & $\begin{array}{c}\text { Barra de Maricá/ } \\
\text { RJ }\end{array}$ & Atlantic Forest & $22^{\circ} 54^{\prime} 00^{\prime \prime} \mathrm{S} / 42^{\circ} 53^{\prime} 23^{\prime \prime} \mathrm{W}$ & $\begin{array}{l}\text { Maia et al. } \\
\text { (2002) }\end{array}$ & Unavailable \\
\hline & Leaf/Fusiform & GM5 & $\begin{array}{c}\text { Lopesia } \\
\text { indaiensis } \\
\text { (Cecidomyiidae) }\end{array}$ & Tamandaré/PE & Atlantic Forest & $8^{\circ} 44^{\prime} 54^{\prime \prime} \mathrm{S} / 35^{\circ} 6^{\prime} 14^{\prime \prime} \mathrm{W}$ & $\begin{array}{l}\text { Maia and de } \\
\text { Oliveira (2018) }\end{array}$ & Unavailable \\
\hline & Leaf/Fusiform & GM5 & $\begin{array}{c}\text { Lopesia } \\
\text { indaiensis } \\
\text { (Cecidomyiidae) }\end{array}$ & Tamandaré /PE & Atlantic Forest & $8^{\circ} 43^{\prime} 30^{\prime \prime} \mathrm{S}, 35^{\circ} 10^{\prime} 40^{\prime \prime} \mathrm{W}$ & $\begin{array}{l}\text { Santos et al. } \\
\text { (2012b) }\end{array}$ & Fig. 3D \\
\hline & Leaf/Fusiform & GM5 & $\begin{array}{c}\text { Lopesia } \\
\text { indaiensis } \\
\text { (Cecidomyiidae) }\end{array}$ & $\begin{array}{l}\text { São Francisco do } \\
\text { Sul/SC }\end{array}$ & Atlantic Forest & $26^{\circ} 17^{\prime} \mathrm{S} / 48^{\circ} 33^{\prime} \mathrm{W}$ & $\begin{array}{l}\text { New record } \\
\text { (Arriola and de } \\
\text { Melo Júnior, } \\
\text { 2016) }\end{array}$ & Fig. 3E \\
\hline & Leaf/Fusiform & GM5 & $\begin{array}{c}\text { Lopesia } \\
\text { indaiensis } \\
\text { (Cecidomyiidae) }\end{array}$ & Cananéia/SP & Atlantic Forest & $25^{\circ} 1^{\prime} 28^{\prime \prime} \mathrm{S} / 47^{\circ} 55^{\prime} 56^{\prime \prime} \mathrm{W}$ & $\begin{array}{l}\text { New record } \\
(\mathrm{MNHN})\end{array}$ & Fig. 3F \\
\hline & Leaf/Fusiform & GM5 & $\begin{array}{c}\text { Lopesia } \\
\text { indaiensis } \\
\text { (Cecidomyiidae) }\end{array}$ & Lençóis/BA & Atlantic Forest & $12^{\circ} 33^{\prime} 47^{\prime \prime} \mathrm{S} / 41^{\circ} 23^{\prime} 24^{\prime \prime} \mathrm{W}$ & $\begin{array}{l}\text { New record } \\
\text { (JBRJ) }\end{array}$ & Fig. 3G \\
\hline & Leaf/Globoid & GM6 & $\begin{array}{l}\text { Asphondylia sp.1 } \\
\text { (Cecidomyiidae) }\end{array}$ & Mangaratiba/RJ & Atlantic Forest & $\begin{array}{c}23^{\circ} 2^{\prime} 34.83^{\prime \prime S} / 43^{\circ} 57^{\prime} 30.80^{\prime \prime W} \\
23^{\circ} 4^{\prime} 4.85^{\prime \prime S} / 43^{\circ} 59^{\prime} 39.41^{\prime \prime W}\end{array}$ & $\begin{array}{l}\text { Rodrigues et al. } \\
\qquad(2014)\end{array}$ & Fig. $3 \mathrm{H}$ \\
\hline & Leaf/Globoid & GM6 & $\begin{array}{l}\text { Asphondylia sp.1 } \\
\text { (Cecidomyiidae) }\end{array}$ & Santa Teresa/ES & Atlantic Forest & $19^{\circ} 09^{\prime} \mathrm{S} / 40^{\circ} 36^{\prime} 00^{\prime \prime} \mathrm{W}$ & $\begin{array}{l}\text { Maia et al. } \\
\text { (2014) }\end{array}$ & Fig. 3I \\
\hline & Leaf/Globoid & GM6 & $\begin{array}{l}\text { Asphondylia sp.1 } \\
\text { (Cecidomyiidae) }\end{array}$ & Bertioga/SP & Atlantic Forest & $23^{\circ} 51^{\prime} 16^{\prime \prime} \mathrm{S} / 46^{\circ} 08^{\prime} 19^{\prime \prime} \mathrm{W}$ & $\begin{array}{l}\text { Maia et al. } \\
\quad(2008)\end{array}$ & Fig. 3J \\
\hline & Leaf/Globoid & GM6 & $\begin{array}{l}\text { Asphondylia sp.1 } \\
\text { (Cecidomyiidae) }\end{array}$ & Tiradentes/MG & Cerrado & $21^{\circ} 03-07^{\prime} \mathrm{S} / 44^{\circ} 06^{-1} 13^{\prime} \mathrm{W}$ & $\begin{array}{l}\text { Maia and } \\
\text { Fernandes } \\
(2004)\end{array}$ & Fig. 3K \\
\hline & Leaf/Globoid & GM6 & $\begin{array}{l}\text { Asphondylia sp.1 } \\
\text { (Cecidomyiidae) }\end{array}$ & $\begin{array}{l}\text { São Francisco do } \\
\text { Sul/SC }\end{array}$ & Atlantic Forest & $26^{\circ} 17^{\prime} \mathrm{S} / 48^{\circ} 33^{\prime} \mathrm{W}$ & $\begin{array}{l}\text { Arriola and de } \\
\text { Melo Júnior } \\
\text { (2016) }\end{array}$ & Fig. 3L \\
\hline & Leaf/Globoid & GM6 & $\begin{array}{l}\text { Meunieriella sp.1 } \\
\text { (Cecidomyiidae) }\end{array}$ & $\begin{array}{l}\text { Araruama, } \\
\text { Arraial do Cabo, } \\
\text { Cabo Frio, São } \\
\text { João da Barra, } \\
\text { Saquarema/RJ }\end{array}$ & Atlantic Forest & 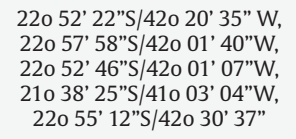 & $\begin{array}{l}\text { Carvalho- } \\
\text { Fernandes et al. } \\
\text { (2016) }\end{array}$ & Fig. 3M \\
\hline & Leaf/Globoid & GM6 & $\begin{array}{l}\text { Meunieriella sp.1 } \\
\text { (Cecidomyiidae) }\end{array}$ & $\begin{array}{l}\text { Barra de Maricá/ } \\
\text { RJ }\end{array}$ & Atlantic Forest & $22^{\circ} 54^{\prime} 00^{\prime} \mathrm{S} / 42^{\circ} 53^{\prime} 23^{\prime \prime} \mathrm{W}$ & $\begin{array}{l}\text { Maia et al. } \\
(2002)\end{array}$ & Unavailable \\
\hline & Stem/Globoid & GM7 & $\begin{array}{l}\text { Undetermined sp } \\
5 \text { (Cecidomyiidae) }\end{array}$ & Tamandaré /PE & Atlantic Forest & $8^{\circ} 43^{\prime} 30^{\prime \prime} \mathrm{S}, 35^{\circ} 10^{\prime} 40^{\prime \prime} \mathrm{W}$ & $\begin{array}{l}\text { Santos et al. } \\
\text { (2012b) }\end{array}$ & Fig. $3 \mathrm{~N}$ \\
\hline & Stem/Fusiform & GM8 & $\begin{array}{l}\text { Undetermined sp } \\
6 \text { (Cecidomyiidae) }\end{array}$ & Santa Teresa/ES & Atlantic Forest & $19^{\circ} 09^{\prime} \mathrm{S} / 40^{\circ} 36^{\prime} 00^{\prime \prime} \mathrm{W}$ & $\begin{array}{l}\text { Maia et al. } \\
(2014)\end{array}$ & Fig. 30 \\
\hline & Stem/Fusiform & GM8 & $\begin{array}{l}\text { Undetermined sp } \\
6 \text { (Cecidomyiidae) }\end{array}$ & Tiradentes/MG & Cerrado & $21^{\circ} 03-07^{\prime} \mathrm{S} / 44^{\circ} 06-13^{\prime} \mathrm{W}$ & $\begin{array}{l}\text { Maia and } \\
\text { Fernandes } \\
(2004)\end{array}$ & Fig. 3P \\
\hline \multirow[t]{7}{*}{$\begin{array}{l}\text { Andira humilis } \\
\text { Mart. ex Benth. }\end{array}$} & Leaf/Fusiform & GM9 & $\begin{array}{l}\text { Lopesia andirae } \\
\text { (Cecidomyiidae) }\end{array}$ & $\begin{array}{l}\text { Chapada dos } \\
\text { Guimarães/MT }\end{array}$ & Cerrado & $15 \cdot 2610 \mathrm{~S} / 055^{\circ} 4723 \mathrm{~W}$ & $\begin{array}{l}\text { Garcia et al. } \\
\text { (2017) }\end{array}$ & Fig. $5 \mathrm{~A}$ \\
\hline & Leaf/Fusiform & GM9 & $\begin{array}{l}\text { Lopesia andirae } \\
\text { (Cecidomyiidae) }\end{array}$ & Barreiras/BA & Cerrado & $\begin{array}{c}11^{\circ} 37^{\prime} \text { and } 12^{\circ} 25^{\prime} \mathrm{S} / 44^{\circ} 34^{\prime} \\
\text { and } 46^{\circ} 23^{\prime} \mathrm{W}\end{array}$ & $\begin{array}{l}\text { Lima and Calado } \\
\text { (2018) }\end{array}$ & Fig. 5B \\
\hline & Leaf/Fusiform & GM9 & $\begin{array}{l}\text { Lopesia andirae } \\
\text { (Cecidomyiidae) }\end{array}$ & Luiz Antônio/SP & Cerrado & $\begin{array}{c}21^{\circ} 30^{\prime} \text { and } 21^{\circ} 40^{\prime} \mathrm{S} / 47^{\circ} 40^{\prime} \\
\text { and } 47^{\circ} 50^{\prime} \mathrm{W}\end{array}$ & $\begin{array}{l}\text { Saito and } \\
\text { Urso-Guimarães } \\
\text { (2012) }\end{array}$ & Fig. 5C \\
\hline & Leaf/Fusiform & GM9 & $\begin{array}{l}\text { Lopesia andirae } \\
\text { (Cecidomyiidae) }\end{array}$ & João Pessoa/PB & Atlantic Forest & 7o 07' 42"S/34o 59' 05”W & $\begin{array}{l}\text { New record } \\
\text { (MNHN) }\end{array}$ & Fig. 5D-E \\
\hline & Leaf/Fusiform & GM9 & $\begin{array}{l}\text { Lopesia andirae } \\
\text { (Cecidomyiidae) }\end{array}$ & Januária/MG & Cerrado & $15^{\circ} 29^{\prime} 15^{\prime \prime} \mathrm{S} / 44^{\circ} 21^{\prime} 40^{\prime \prime} \mathrm{W}$ & $\begin{array}{c}\text { New record } \\
\text { (Luz et al., 2012) }\end{array}$ & Fig. $5 \mathrm{~F}$ \\
\hline & Leaf/Fusiform & GM9 & $\begin{array}{l}\text { Lopesia andirae } \\
\text { (Cecidomyiidae) }\end{array}$ & Caldas/MG & Cerrado & $21055^{\prime} 25^{\prime \prime} \mathrm{S} / 460$ 23' $10^{\prime \prime} \mathrm{W}$ & $\begin{array}{l}\text { New record } \\
(\text { MNHN) }\end{array}$ & Fig. 5G \\
\hline & Leaf/Fusiform & GM9 & $\begin{array}{l}\text { Lopesia andirae } \\
\text { (Cecidomyiidae) }\end{array}$ & Prata/MG & Cerrado & $19^{\circ} 17^{\prime} 56^{\prime \prime} \mathrm{S} / 48^{\circ} 54^{\prime} 51^{\prime \prime} \mathrm{W}$ & New record (NY) & Fig. $5 \mathrm{H}$ \\
\hline
\end{tabular}


Table 1

Continued...

\begin{tabular}{|c|c|c|c|c|c|c|c|c|}
\hline Host plant & $\begin{array}{c}\text { Organ/ } \\
\text { Morphotype }\end{array}$ & $\begin{array}{l}\text { Number of gall } \\
\text { morphotype } \\
\text { (GM) }\end{array}$ & Gall inducer & $\begin{array}{l}\text { Municipality/ } \\
\text { State }\end{array}$ & $\begin{array}{l}\text { Phytogeographic } \\
\text { domain }\end{array}$ & Coordinates & Reference & Figure \\
\hline \multirow[t]{5}{*}{$\begin{array}{l}\text { Andira nitida } \\
\text { Mart. ex Benth. }\end{array}$} & Leaf/Lenticular & GM10 & $\begin{array}{l}\text { Undetermined sp } \\
7 \text { (Cecidomyiidae) }\end{array}$ & Guarapari/ES & Atlantic Forest & $\begin{array}{c}20^{\circ} 33^{\prime}-20^{\circ} 38^{\prime} \mathrm{S} / 40^{\circ} 23^{\prime}-40^{\circ} \\
26^{\prime} \mathrm{W}\end{array}$ & $\begin{array}{l}\text { Bregonci et al. } \\
\text { (2010) }\end{array}$ & Fig. 7A \\
\hline & Leaf/Lenticular & GM10 & $\begin{array}{l}\text { Undetermined sp } \\
7 \text { (Cecidomyiidae) }\end{array}$ & Vila Velha/ES & Atlantic Forest & $20^{\circ} 27^{\prime} 44.0^{\prime \prime} \mathrm{S} / 40^{\circ} 20^{\prime} 36.0^{\prime \prime} \mathrm{W}$ & $\begin{array}{l}\text { New record } \\
\text { (MBLB) }\end{array}$ & Fig. 7B \\
\hline & Leaf/Lenticular & GM10 & $\begin{array}{l}\text { Undetermined sp } \\
7 \text { (Cecidomyiidae) }\end{array}$ & Prado/BA & Cerrado & $17^{\circ} 16^{\prime} 20.0^{\prime \prime} \mathrm{S} / 39^{\circ} 14^{\prime} 30.0^{\prime \prime} \mathrm{W}$ & $\begin{array}{l}\text { New record } \\
\quad(\text { UEC) }\end{array}$ & Fig. 7C \\
\hline & Leaf/Marginal roll & GM11 & $\begin{array}{l}\text { Undetermined sp } \\
8 \text { (Cecidomyiidae) }\end{array}$ & Guarapari/ES & Atlantic Forest & $\begin{array}{c}20^{\circ} 33^{\prime}-20^{\circ} 38^{\prime} \mathrm{S} / 40^{\circ} 23^{\prime}-40^{\circ} \\
26^{\prime} \mathrm{W}\end{array}$ & $\begin{array}{l}\text { Bregonci et al. } \\
\quad(2010)\end{array}$ & Fig. 7D \\
\hline & Leaf/Globoid & GM12 & $\begin{array}{l}\text { Undetermined sp } \\
9 \text { (Cecidomyiidae) }\end{array}$ & Guarapari/ES & Atlantic Forest & $\begin{array}{c}20^{\circ} 33^{\prime}-20^{\circ} 38^{\prime} \mathrm{S} / 40^{\circ} 23^{\prime}-40^{\circ} \\
26^{\prime} \mathrm{W}\end{array}$ & $\begin{array}{l}\text { Bregonci et al. } \\
\quad(2010)\end{array}$ & Fig. 7E \\
\hline $\begin{array}{c}\text { Andira } \\
\text { surinamensis } \\
\text { (Bondt) Splitg. ex } \\
\text { Amshoff }\end{array}$ & Leaf/Cylindrical & GM13 & $\begin{array}{c}\text { Undetermined } \\
\text { sp } 10 \\
\text { (Cecidomyiidae) }\end{array}$ & $\begin{array}{c}\text { Porto Trombetas/ } \\
\text { PA }\end{array}$ & Amazonia & $1^{\circ} 40^{\prime} \mathrm{S} / 56^{\circ} 27^{\prime} \mathrm{W}$ & $\begin{array}{l}\text { Almada et al. } \\
\qquad(2011)\end{array}$ & Fig. 8B \\
\hline \multirow[t]{13}{*}{$\begin{array}{l}\text { Andira vermifuga } \\
\text { (Mart.) Benth. }\end{array}$} & Leaf/Fusiform & GM14 & $\begin{array}{l}\text { Undetermined } \\
\text { sp } 11 \\
\text { (Cecidomyiidae) }\end{array}$ & Caldas Novas/GO & Cerrado & $17^{\circ} 42^{\prime} 39^{\prime \prime} \mathrm{S} / 48^{\circ} 38^{\prime} 27^{\prime \prime} \mathrm{W}$ & $\begin{array}{l}\text { Santos et al. } \\
\text { (2012a) }\end{array}$ & Unavailable \\
\hline & Leaf/Laminar & GM15 & $\begin{array}{l}\text { Undetermined } \\
\text { sp } 12 \\
\text { (Cecidomyiidae) }\end{array}$ & Caldas Novas/GO & Cerrado & $17^{\circ} 42^{\prime} 39^{\prime \prime} \mathrm{S} / 48^{\circ} 38^{\prime} 27^{\prime \prime} \mathrm{W}$ & $\begin{array}{l}\text { Santos et al. } \\
\text { (2012a) }\end{array}$ & Fig. 10A \\
\hline & Leaf/Globoid & GM16 & $\begin{array}{l}\text { Undetermined } \\
\text { sp 13? } \\
\text { (Cecidomyiidae) }\end{array}$ & Caldas Novas/GO & Cerrado & $17^{\circ} 42^{\prime} 39^{\prime \prime} \mathrm{S} / 48^{\circ} 38^{\prime} 27^{\prime \prime} \mathrm{W}$ & $\begin{array}{l}\text { Santos et al. } \\
\text { (2012a) }\end{array}$ & Unavailable \\
\hline & Leaf/Globoid & GM17 & $\begin{array}{c}\text { Lopesia } \\
\text { chapadensis } \\
\text { (Cecidomyiidae) }\end{array}$ & $\begin{array}{l}\text { Chapada dos } \\
\text { Guimarães/MT }\end{array}$ & Cerrado & $15.4093^{\circ} \mathrm{S} / 55.8330^{\circ} \mathrm{W}$ & $\begin{array}{l}\text { Garcia and } \\
\text { Urso-Guimarães } \\
\text { (2018) }\end{array}$ & Fig. 10B \\
\hline & Leaf/Globoid & & $\begin{array}{c}\text { Lopesia } \\
\text { chapadensis } \\
\text { (Cecidomyiidae) }\end{array}$ & Ipameri/GO & Cerrado & $17^{\circ} 43^{\prime} 00.0^{\prime \prime} \mathrm{S} / 48^{\circ} 09^{\prime} 00.0^{\prime \prime} \mathrm{W}$ & $\begin{array}{l}\text { New record } \\
\quad(\text { CEN })\end{array}$ & Fig. 10C \\
\hline & Leaf/Globoid & GM17 & $\begin{array}{c}\text { Lopesia } \\
\text { chapadensis } \\
\text { (Cecidomyiidae) }\end{array}$ & $\begin{array}{l}\text { Alvorada do } \\
\text { Norte/GO }\end{array}$ & Cerrado & $14^{\circ} 30^{\prime} 41^{\prime \prime} \mathrm{S} / 46^{\circ} 46^{\prime} 14^{\prime \prime} \mathrm{W}$ & $\begin{array}{l}\text { New record } \\
\text { (JBRJ) }\end{array}$ & Fig. 10D \\
\hline & Leaf/Globoid & GM17 & $\begin{array}{c}\text { Lopesia } \\
\text { chapadensis } \\
\text { (Cecidomyiidae) }\end{array}$ & Brasília/DF & Cerrado & $15^{\circ} 46^{\prime} 46.9^{\prime \prime} \mathrm{S} / 47^{\circ} 55^{\prime} 46.9^{\prime \prime} \mathrm{W}$ & New record (NY) & Fig. 10E \\
\hline & Leaf/Globoid & GM17 & $\begin{array}{c}\text { Lopesia } \\
\text { chapadensis } \\
\text { (Cecidomyiidae) }\end{array}$ & Missão Velha/CE & Cerrado & $7^{\circ} 10^{\prime} 54^{\prime \prime} \mathrm{S} / 3^{\circ} 4^{\prime} 3^{\prime \prime} \mathrm{W}$. & $\begin{array}{l}\text { New record } \\
\text { (JBRJ) }\end{array}$ & Fig. 10F \\
\hline & Leaf/Lenticular1 & GM18 & $\begin{array}{c}\text { Undetermined } \\
\text { sp } 14 \\
\text { (Cecidomyiidae) }\end{array}$ & Caldas Novas/GO & Cerrado & $17^{\circ} 42^{\prime} 39^{\prime \prime} \mathrm{S} / 48^{\circ} 38^{\prime} 27^{\prime \prime} \mathrm{W}$ & $\begin{array}{l}\text { Santos et al. } \\
\text { (2012a) }\end{array}$ & Unavailable \\
\hline & Leaf/Lenticular 2 & GM19 & $\begin{array}{c}\text { Undetermined } \\
\text { sp } 15 \\
\text { (Cecidomyiidae) }\end{array}$ & Goiânia/GO & Cerrado & $16^{\circ} 38^{\prime} 11.79^{\prime \prime} \mathrm{S} / 48^{\circ} 39^{\prime} 50.82^{\prime \prime} \mathrm{W}$ & $\begin{array}{l}\text { Bergamini et al. } \\
\text { (2017) }\end{array}$ & Fig. 10G \\
\hline & Leaf/Lenticular 2 & GM19 & $\begin{array}{c}\text { Undetermined } \\
\text { sp } 15 \\
\text { (Cecidomyiidae) }\end{array}$ & $\begin{array}{l}\text { Nova Xavantina/ } \\
\text { MT }\end{array}$ & Cerrado & $14^{\circ} 40^{\prime} 23.9^{\prime \prime} \mathrm{S} / 52^{\circ} 21^{\prime} 11.2^{\prime \prime} \mathrm{W}$ & $\begin{array}{l}\text { New record } \\
\text { (JBRJ) }\end{array}$ & Fig. $10 \mathrm{H}$ \\
\hline & Leaf/Lenticular 3 & GM20 & $\begin{array}{c}\text { Undetermined } \\
\text { sp } 16 \\
\text { (Cecidomyiidae) }\end{array}$ & Goiânia/GO & Cerrado & $16^{\circ} 38^{\prime} 11.79^{\prime \prime} \mathrm{S} / 48^{\circ} 39^{\prime} 50.82^{\prime \prime} \mathrm{W}$ & $\begin{array}{l}\text { Bergamini et al. } \\
\text { (2017) }\end{array}$ & Fig. 10I \\
\hline & Leaf/Lenticular 3 & GM20 & $\begin{array}{c}\text { Undetermined } \\
\text { sp } 16 \\
\text { (Cecidomyiidae) }\end{array}$ & Niquelândia/GO & Cerrado & $\begin{array}{c}14^{\circ} 31^{\prime} 14^{\prime \prime S}-14^{\circ} 31^{\prime} 14^{\prime \prime} \mathrm{S} / 48^{\circ} \\
9^{\prime} 8^{\prime \prime} \mathrm{W}-48^{\circ} 9^{\prime} 8^{\prime \prime}\end{array}$ & New record (K) & Fig. 10J \\
\hline
\end{tabular}

of this species, all induced by unidentified species of Cecidomyiidae. Araújo et al. (2014) found them in the Parque Nacional de Emas located in Mineiros, South of State of Goiás (Fig. 1). The absence of morphotype figures in the original inventory did not allow the comparison with literature or exsiccates and, consequently, no new records were found.

Andira fraxinifolia Benth. has A. parvifolia Mart. ex Benth. as a synonym and both names were searched for association with insect galls. The species is widely distributed in Central and East coast region in Caatinga, Cerrado, and Atlantic Forest phytogeographic domains (Ramos et al., 2020) (Fig. 2).

Review of literature presented four morphotypes: fusiform and globoid exclusively in leaves and globoid and fusiform in stems. Lopesia indaiensis (Cecidomyiidae) is the inducer of fusiform leaf galls in A. fraxinifolia, originally recorded in Minas Gerais (Dores do
Indaiá) in Cerrado and in Pernambuco (Tamandaré), in Rio de Janeiro (Mangaratiba), and in São Paulo (Bertioga) in Atlantic Forest (Maia and de Oliveira, 2018) (Fig. 3A-C). Additional records of the fusiform morphotype (Fig. 3D-G) and its gall inducer were found in São Paulo (Cananéia), in Bahia (Lençóis), and in São Francisco do Sul/SC (Arriola and de Melo Júnior, 2016) (Fig. 2). For the other morphotypes (Fig. 3H-P), there is no confirmation of the gall inducer species, so we presented the details about them in Table 1.

Andira humilis is widely distributed in all regions of Brazil, present in the phytogeographic domains of Amazonia, Caatinga, and Cerrado (Ramos et al., 2020) (Fig. 4). The only morphotype recorded to $A$. humilis is fusiform, green with placement in the adaxial surface of leaves (Fig. 5). The gall is induced by Lopesia andirae (Cecidomyiidae) originally recorded to Chapada dos Guimarães/MT, Barreiras/BA, and Luiz 


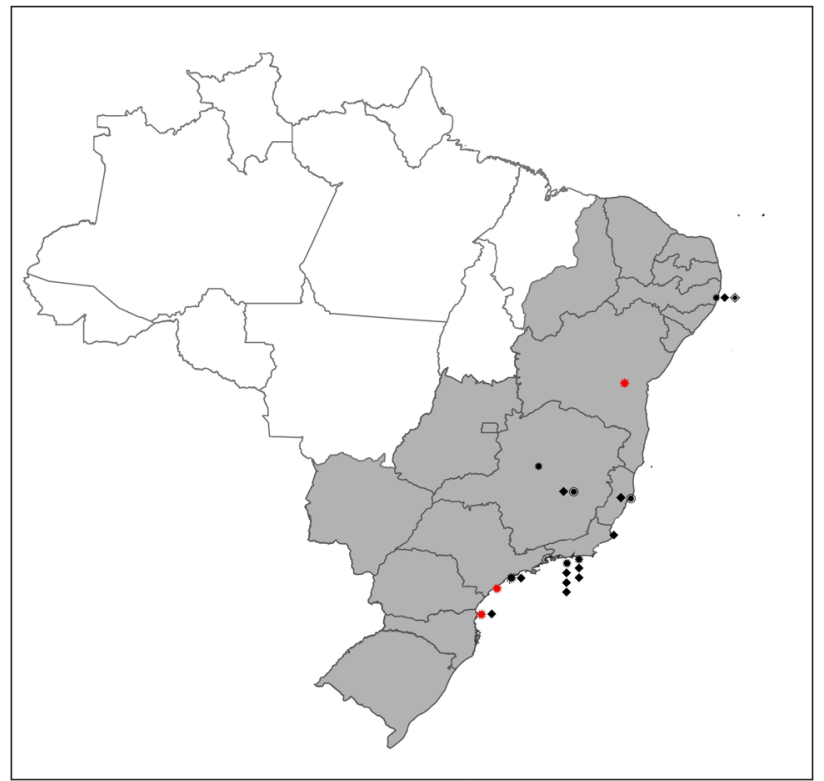

\section{Occurrence of Andira fraxinifolia}

Benth. in Brazilian states

\section{$\square$ No occurrence \\ $\square$ Confirmed occurrence}

Gall morphotypes in Andira fraxinifolia Benth. in Brazilian states

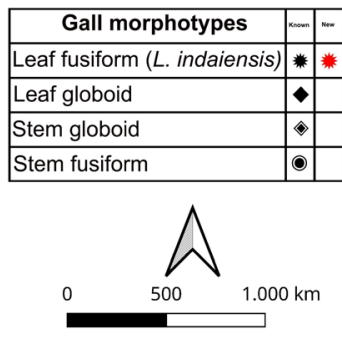

Figure 2 Distribution of Andira fraxinifolia Benth. in Brazilian states and the points of occurrence of the gall morphotypes induced in the species.
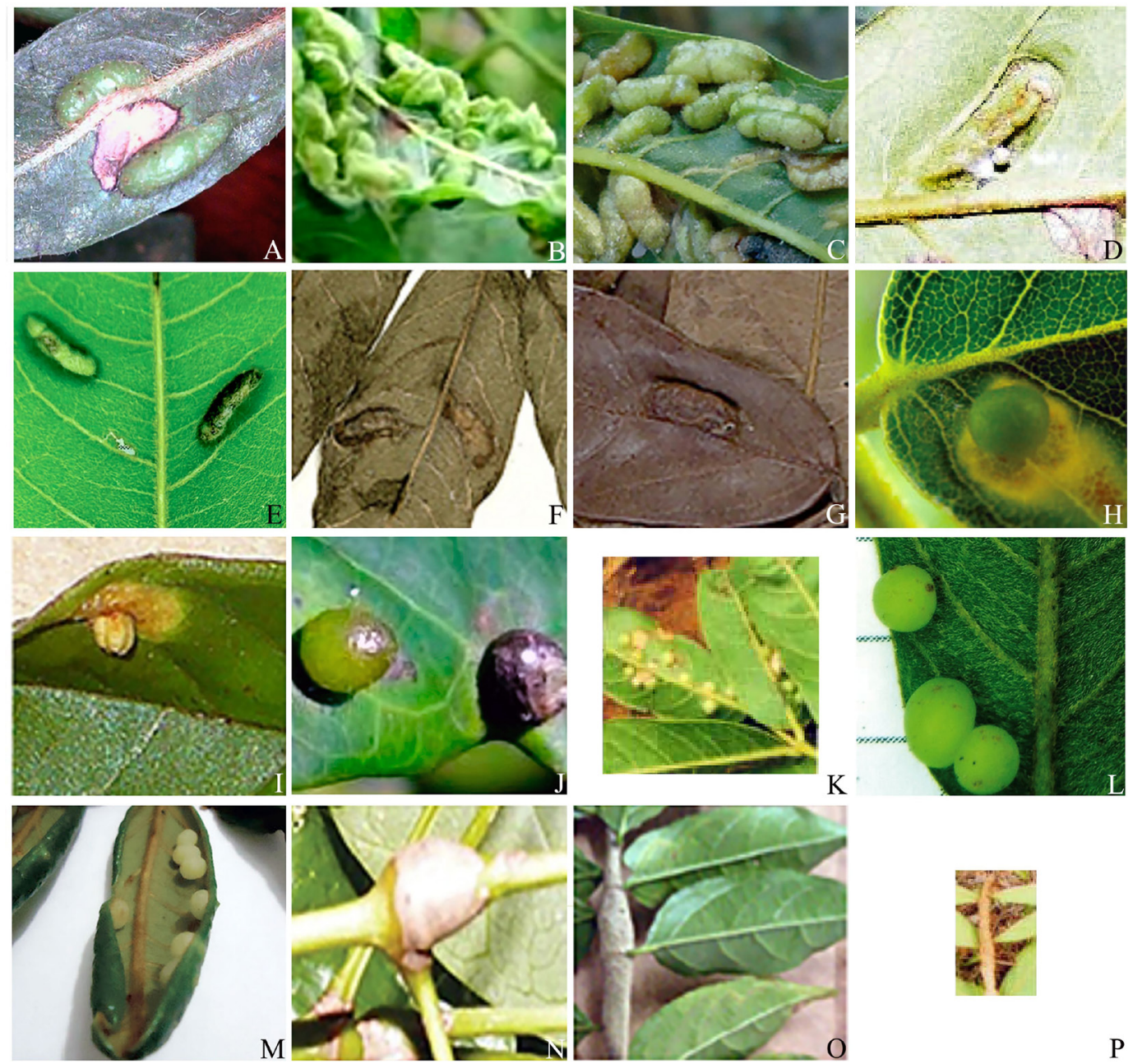

Figure 3 Morphotypes of insect galls in Andira fraxinifolia Benth. from several localities. A-G. Fusiform leaf galls, A. Dores do Indaiá/MG (Maia and de Oliveira, 2018); B. Bertioga/ SP (Maia et al., 2008); C. Mangaratiba/RJ (Rodrigues et al., 2014); D. Tamandaré/PE (Santos et al., 2012b); E. São Francisco do Sul/SC (Arriola and de Melo Júnior, 2016); F. Exsiccate from Cananéia/SP (Photo: D. A. Grande et al.); G. Exsiccate from Lencóis/BA (Photo: E. Pereira, 1956); H- M. Globoid leaf galls, H. Mangaratiba/RJ (Rodrigues et al., 2014); I. Santa Teresa/ES (Maia et al., 2014); J. Bertioga/SP (Maia et al., 2008); K. Tiradentes/MG (Maia and Fernandes, 2004); L. São Francisco do Sul/SC (Arriola and de Melo Júnior, 2016); M. Araruama, Arraial do Cabo, Cabo Frio, São João da Barra, Saquarema/RJ (Carvalho-Fernandes et al., 2016); N. Globoid stem gall from Tamandaré/PE (Santos et al., 2012b); O. Fusiform stem gall from Santa Teresa/ES (Maia et al., 2014); P. Tiradentes/MG (Maia and Fernandes, 2004). 


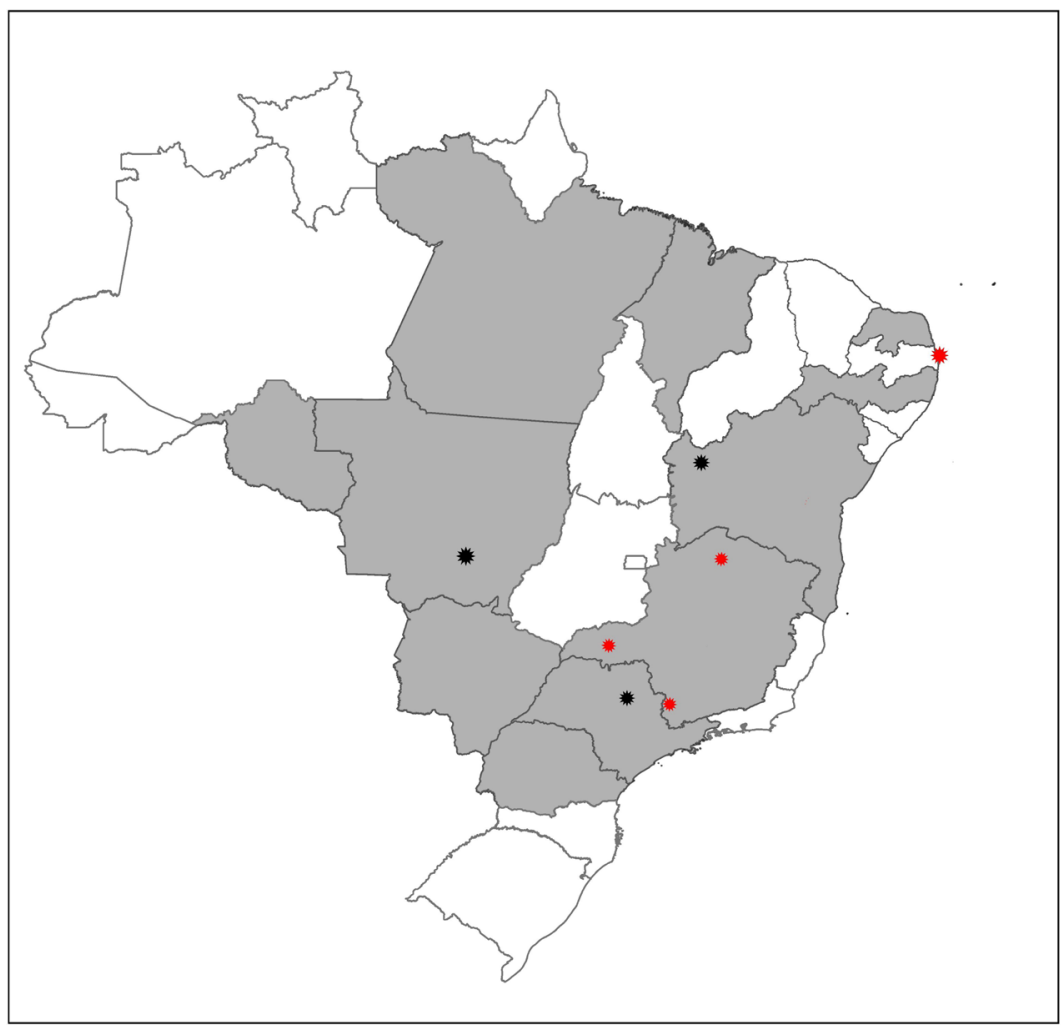

\section{Occurrence of Andira humilis Mart. ex Benth. in Brazilian states}

No occurrence

Confirmed occurrence

\section{Gall morphotype in Andira humilis Mart. ex Benth. in Brazilian states}

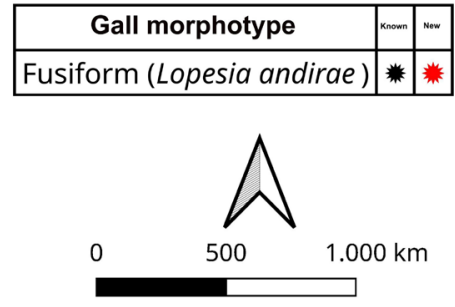

Figure 4 Distribution of Andira humilis Mart. ex Benth. in Brazilian states and the points of occurrence of the gall morphotypes induced in the species.
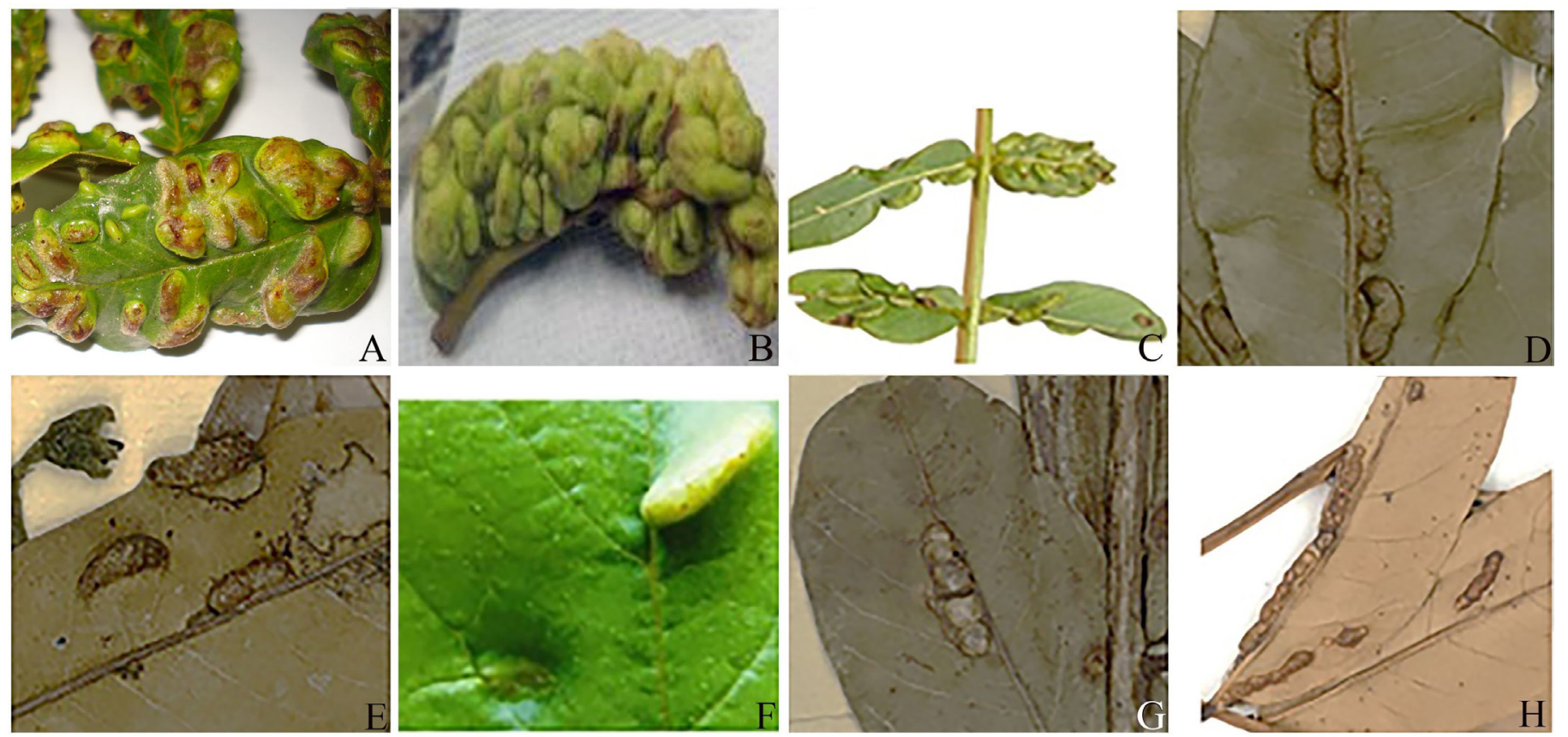

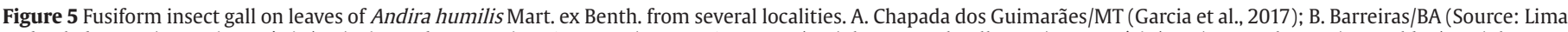

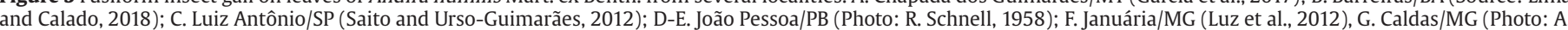
F. Regnell); H. Prata/MG (Photo: R. Caborial, 1949).

Antônio/SP (Garcia et al., 2017). New records were obtained in digital herbaria to Caldas/MG, Prata/MG, and João Pessoa/PB and from literature to Januária/MG (Luz et al., 2012), (Fig. 4). This is the first record of the fusiform morphotype in $A$. humilis to the Paraíba state. Through the search tool in virtual herbaria it was possible to verify a new distribution point in northeastern Brazil, corroborating the hypothesis of Lima and Calado (2020) that there was suitability habitat for the occurrence of Lopesia andirae in areas of Cerrado, Caatinga, and Atlantic Forest in 


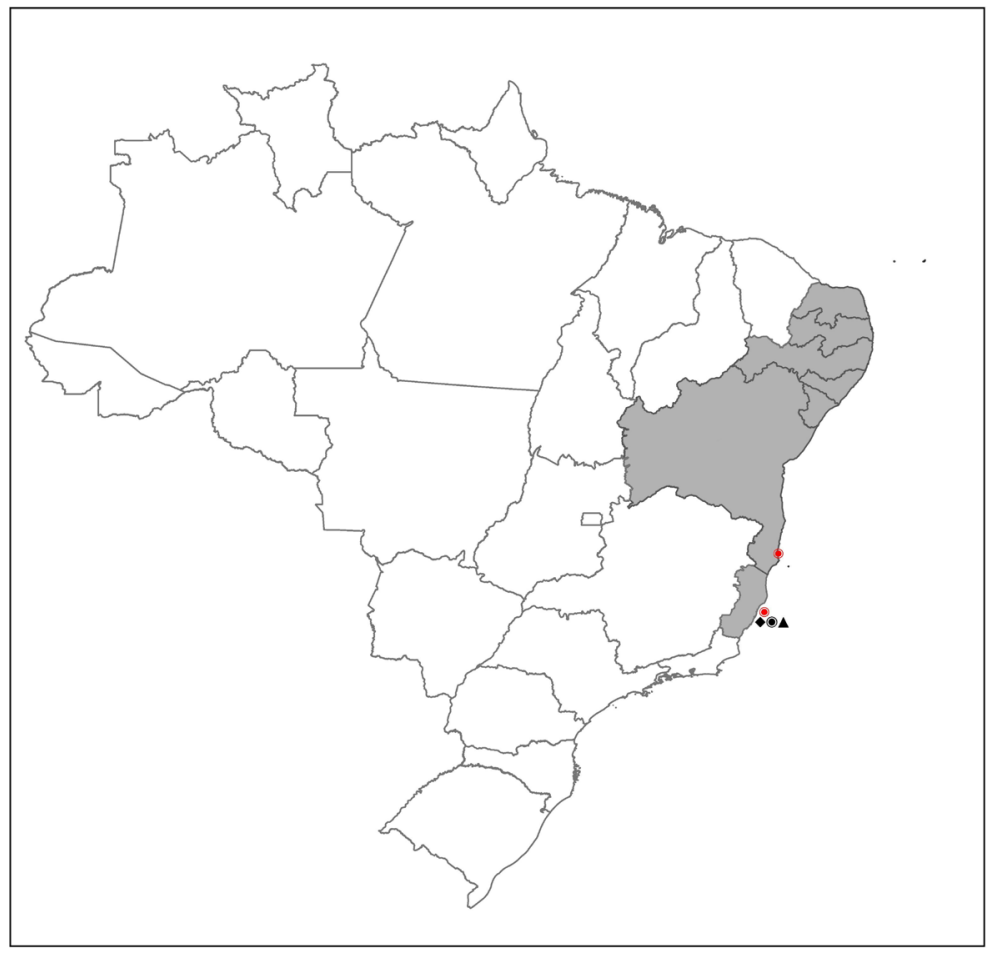

Occurrence of in Andira nitida Mart. ex Benth. in Brazilian states

No occurrence

Confirmed occurrence

\section{Gall morphotypes in Andira nitida Mart. ex Benth. in Brazilian states}

\begin{tabular}{|l|c|c|}
\hline \multicolumn{1}{|c|}{ Gall morphotypes } & known & Now \\
\hline Leaf lenticular & 0 & 0 \\
\hline Leaf marginal roll & $\mathbf{\Delta}$ & \\
\hline Leaf globoid & $\bullet$ & \\
\hline
\end{tabular}

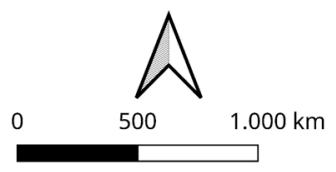

Figure 6 Distribution of Andira nitida Mart. ex Benth. in Brazilian states and the points of occurrence of the gall morphotypes induced in the species.
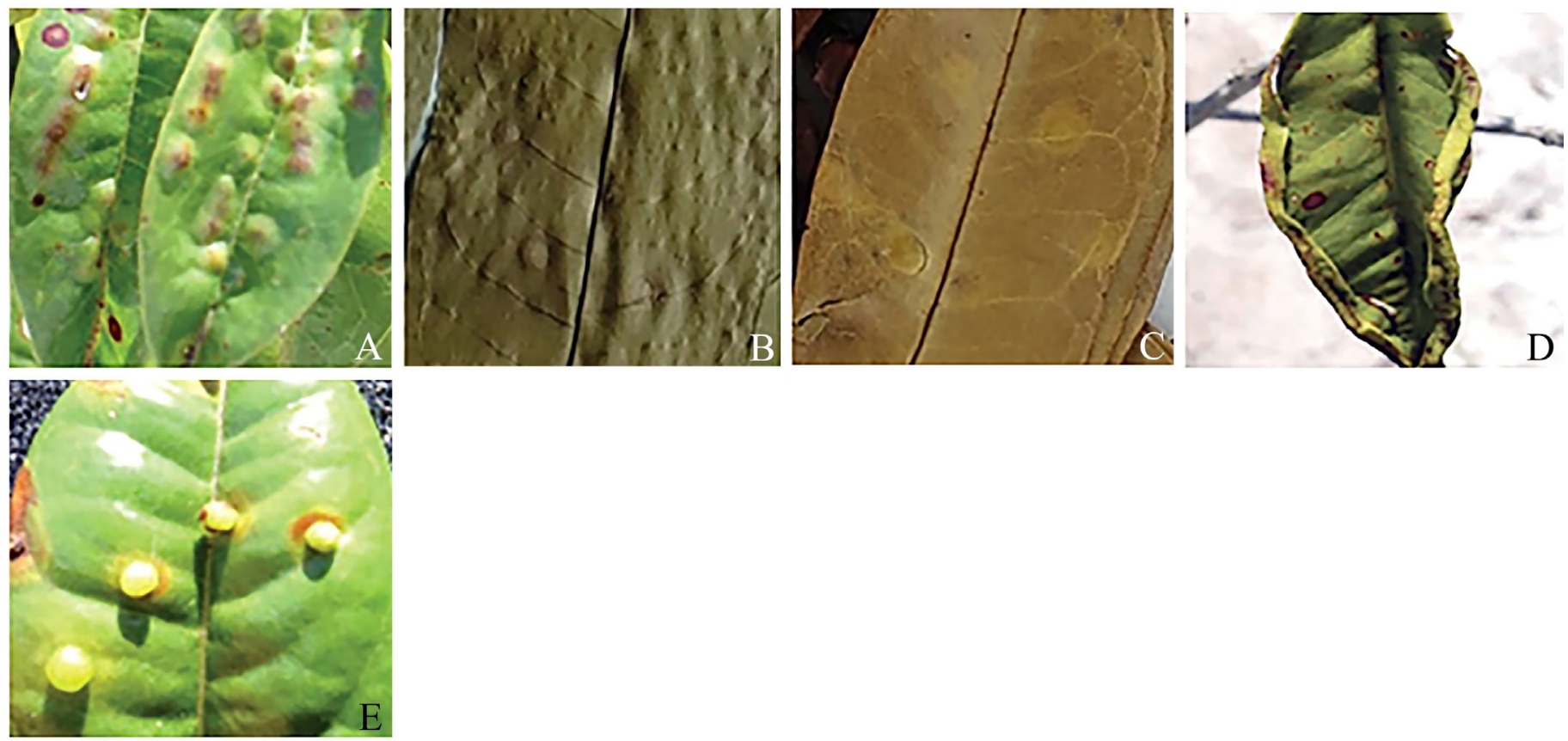

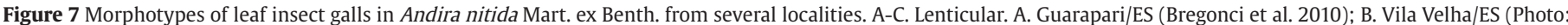
F.A.R. Matos et al., 2006); C. Prado/BA (Photo: D.M. Neves et al., 2014); D. Marginal roll. Guarapari/ES (Bregonci et al. 2010); E. Globoid. Guarapari/ES (Bregonci et al. 2010).

Brazil. This new register of the gall morphotype allow us to extend the distribution of the Lopesia andirae from the north of São Paulo State to, at least, the Atlantic Forest region on the coast of Paraíba State and the Central region of the State of Mato Grosso.

Andira nitida Mart. ex Benth. is distributed in the coastal regions from the north of the State of Espírito Santo to Rio Grande do Norte, in the phytogeographic domain of Atlantic Forest (Ramos et al., 2020) (Fig. 6). The morphotypes registered in the literature were lenticular, marginal roll, and globoid on leaves (Fig. 7), induced by undetermined species of Cecidomyiidae family from Guarapari/ES (Bregonci et al., 2010). New records to the gall were found in digital herbaria to Prado/ BA and Vila Velha/ES (Fig. 6). This is the first record to the lenticular morphotype in A. nitida to the Bahia state.

Andira surinamensis is distributed in the Central-North regions of Brazil in the Amazonia, Caatinga, and Cerrado phytogeographic domains (Pennington, 2003; Ramos et al., 2020) (Fig. 8A). One cylindrical 

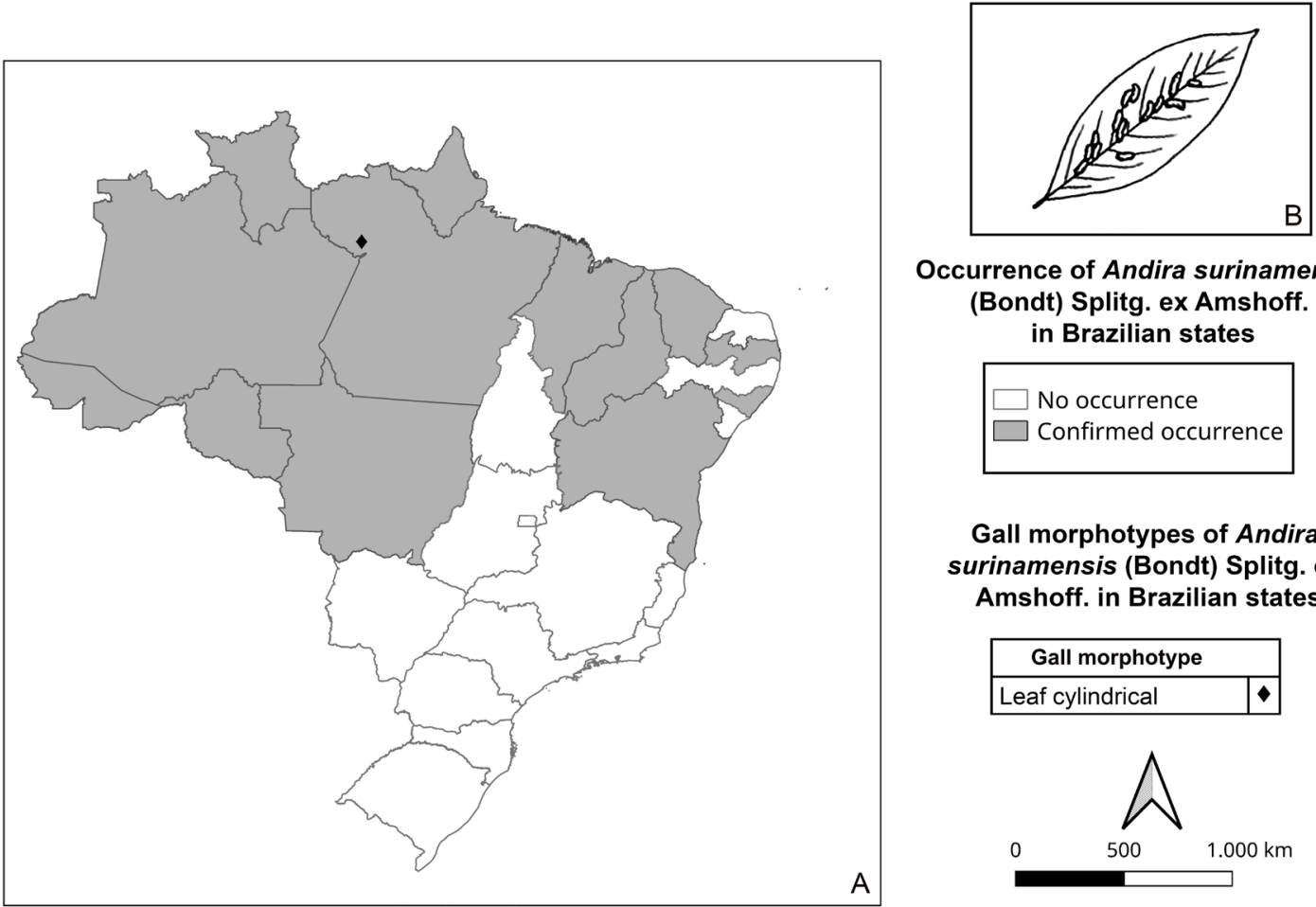

Occurrence of Andira surinamensis (Bondt) Splitg. ex Amshoff. in Brazilian states

\section{No occurrence}

Confirmed occurrence

Gall morphotypes of Andira surinamensis (Bondt) Splitg. ex Amshoff. in Brazilian states

\begin{tabular}{|l|l|}
\hline \multicolumn{1}{|c|}{ Gall morphotype } \\
\hline Leaf cylindrical \\
\hline
\end{tabular}

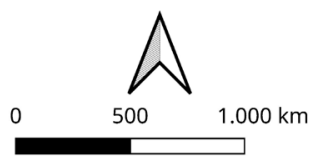

Figure 8 A. Distribution of Andira surinamensis (Bondt) Splitg. ex Amshoff in Brazilian states and the point of occurrence of the gall morphotype induced in the species. B. Cylindrical leaf gall in A. surinamensis found in Porto Trombetas/PA (Almada et al., 2011).

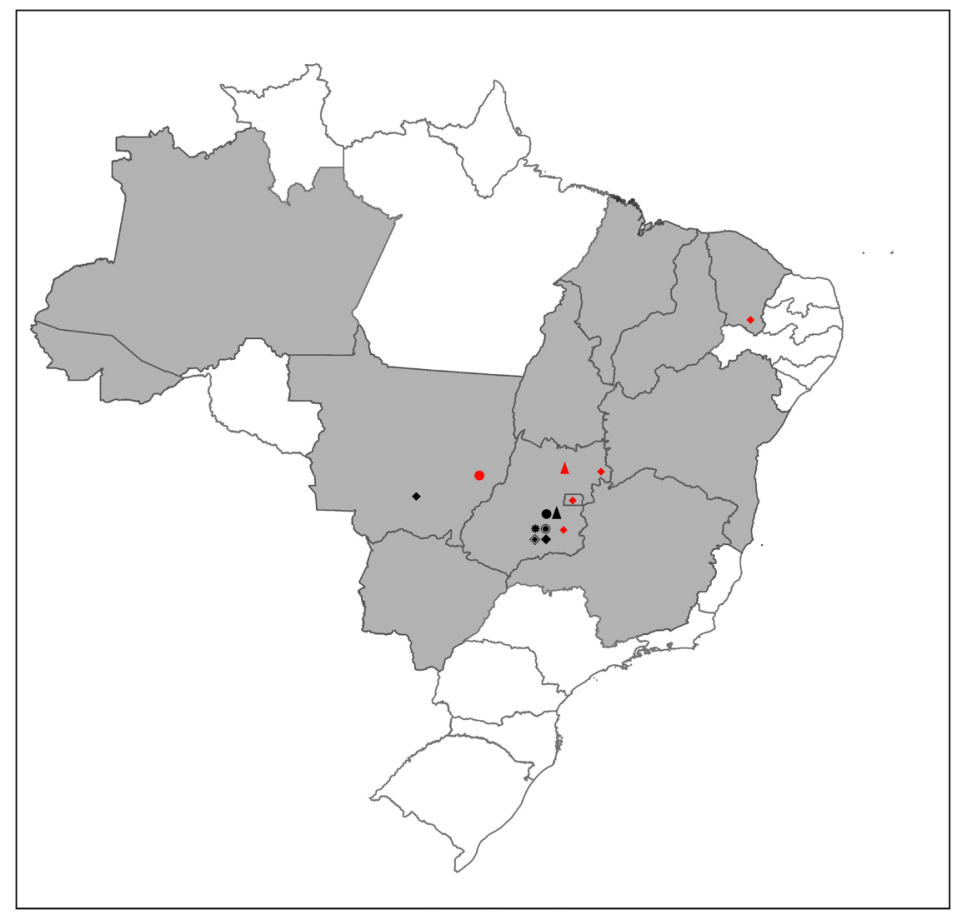

Occurrence of Andira vermifuga (Mart.) Benth. in Brazilian states

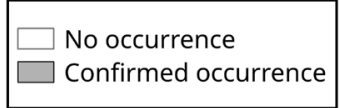

Gall morphotypes in Andira vermifuga (Mart.) Benth. in Brazilian states

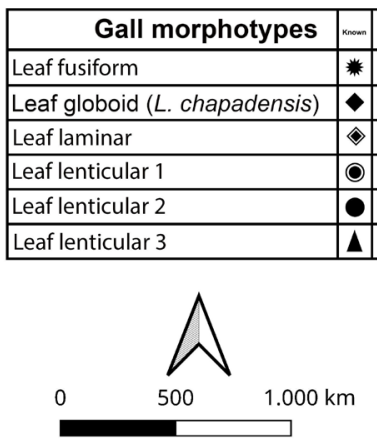

Figure 9 Distribution of Andira vermifuga (Mart.) ex Benth. in Brazilian states and the points of occurrence of the gall morphotypes induced in the species.

morphotype was recorded on A. surinamensis leaves in Brazil (Fig. 8B) in Porto Trombetas/PA (Almada et al., 2011). The gall inducer is not determined, and no similar gall was found in the examined exsiccates. Further studies are needed to understand the geographical limits of this morphotype, as well as to study the life cycle and identity of the gall inducer.
Andira vermifuga is synonym of $A$. paniculata Benth and both names were searched for association with insect galls. The species is distributed in all regions of Brazil, except for South in the phytogeographic domains of Amazonia, Caatinga, Cerrado, and Atlantic Forest (Ramos et al., 2020) (Fig. 9). Although there are many references to the galls in $A$. vermifuga, most inducers are unknown at the species level. Six leaf morphotypes 

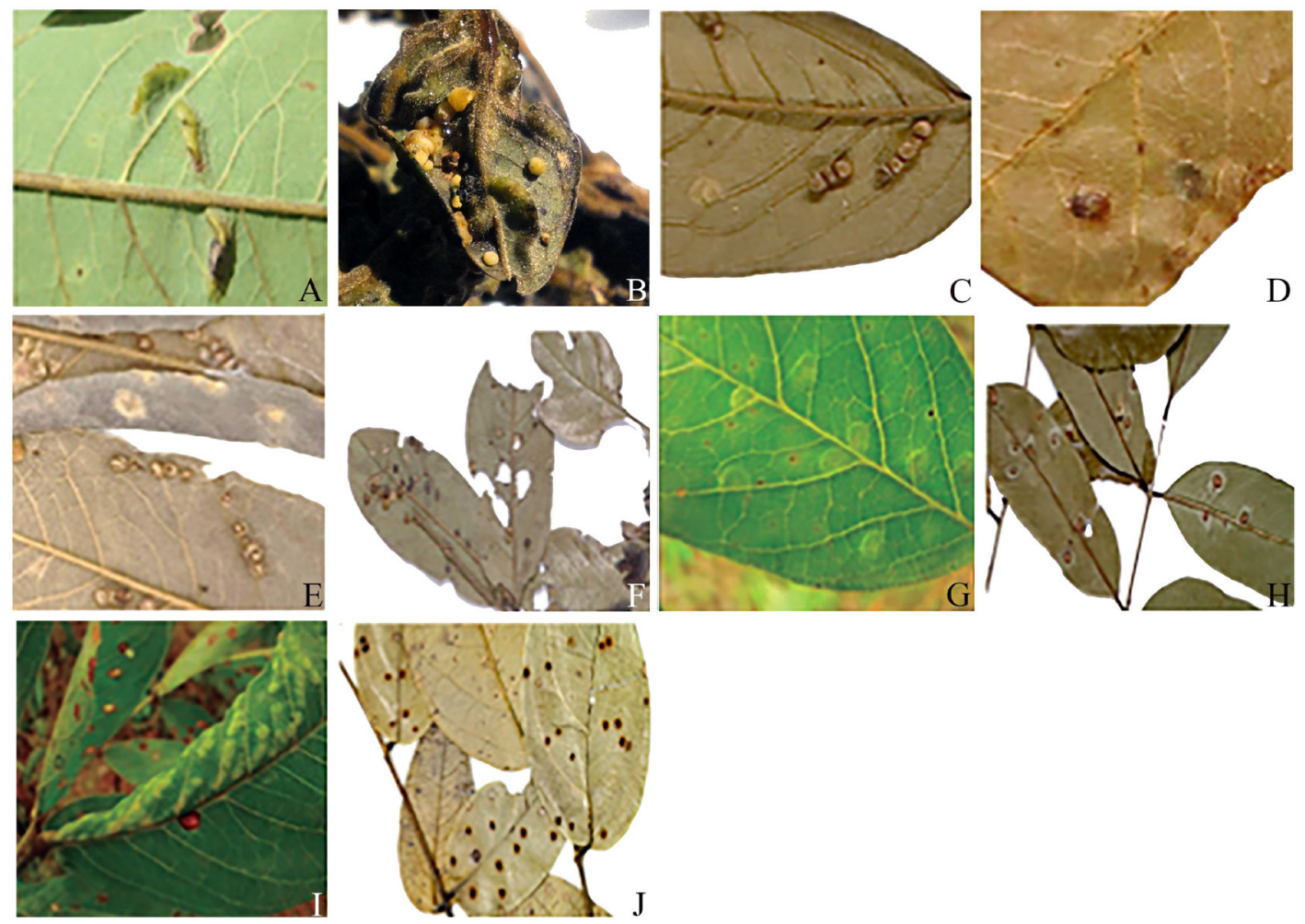

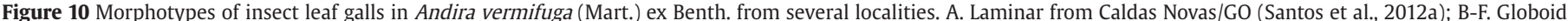

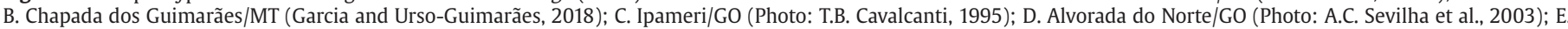

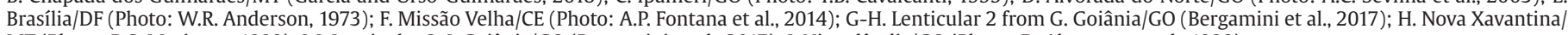
MT (Photo: B.S. Marimon, 1999); I-J. Lenticular 3. I. Goiânia/GO (Bergamini et al., 2017); J. Niquelândia/GO (Photo: D. Alvarenga et al., 1998).
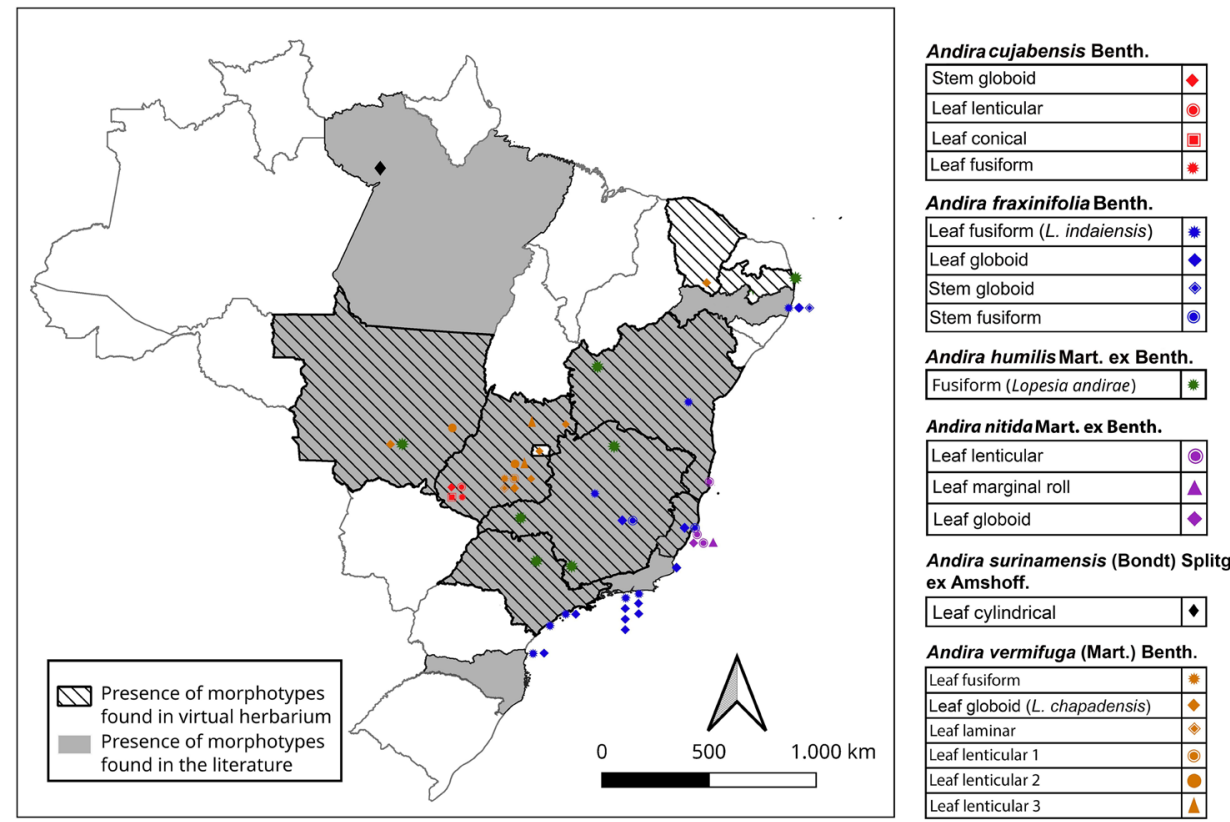

Figure 11 Geographical distribution of the Cecidomyiidae species (Diptera) and gall morphotypes induced in Andira Lam. (Fabaceae) in Brazil.

are described: fusiform, laminar, three types of lenticular, and globoid (Santos et al., 2012a; Bergamini et al., 2017) (Fig. 10). One gall inducer is described for the globoid morphotype (Fig. 10B), Lopesia chapadensis, from Chapada dos Guimarães/MT. The other is identified only at family level and also belongs to Cecidomyiidae. New records were found for the globoid morphotype from Ipameri/GO, Alvorada do Norte/GO, Missão Velha/CE, and Brasília/DF (Fig. 10C-F), and to the lenticular from Nova Xavantina/MT and Niquelândia/ GO (Fig. 10H, 10J).

\section{Discussion}

From 49 records of 20 distinct morphotypes of insect galls in Andira, 24 are referred as cecidomyiids, with three of them identified in species level. A map with the synthesis of all records of the gall inducers in Andira species is presented in the Figure 11. The fusiform morphotype of $A$. fraxinifolia induced by Lopesia indaiensis (Cecidomyiidae) has an expansion of distribution from four to six states (new records to the 
states of Bahia and Santa Catarina), and three new sampling points (São Francisco do Sul/SC, Cananéia/SP, and Lençóis/BA). In A. fraxinifolia, the leaf globoid gall induced by an unidentified species of Asphondylia has an inquiline, Meunieriella sp., both from Cecidomyiidae family was recorded to the states of Rio de Janeiro, Espírito Santo, São Paulo, Minas Gerais, and Santa Catarina, but further studies are needed to confirm the identity of the inductor.

The fusiform morphotype of $A$. humilis induced by Lopesia andirae (Cecidomyiidae) was recorded in the states of Bahia (Lima and Calado, 2018), Mato Grosso (Garcia et al., 2017), and São Paulo (Saito and UrsoGuimarães, 2012). Five new records were found, three in the state of Minas Gerais (Luz et al., 2012) and two in Paraíba (exsiccates).

Three leaf morphotypes were registered to Andira nitida in Guarapari, State of Espírito Santo, induced by undetermined species of cecidomyiids (Bregonci et al., 2010): lenticular, marginal roll and globoid. Two new registers were found in exsiccates to the lenticular morphotype: one in Espírito Santo State (Vila Velha) and the other in Bahia State (Prado).

From the six morphotypes of galls in A. vermifuga, we found new registers to three of them, the globoid one and two lenticulars. The globoid leaf gall induced by Lopesia chapadensis has four new records, two to Goiás State (Alvorada do Norte and Ipameri), one to Ceará (Missão Velha), and one to the Federal District (Brasília). The previous distribution included only the type-locality, in the Mato Grosso State (Garcia and Urso-Guimarães, 2018). Two lenticular types found originally only in Goiânia (Goiás State), gained new records: the type 2 to Nova Xavantina, Mato Grosso State, and the type 3 to Niquelândia (Goiás State).

Most of the morphotypes found in these species induced galls in leaves. This is a widely reported pattern in the literature (Mani, 1964; Maia et al., 2008; Saito and Urso-Guimarães, 2012; Santos-Silva and Araújo, 2020) found in all Brazilian regions (Araújo et al., 2019) and also recovered in different biogeographic regions (Stone and Schönrogge, 2003; Veldtman and McGeoch, 2003; Cuevas-Reyes et al., 2004). This pattern is attributed to the abundance and constancy of leaves in plants, besides the continuous flow of nutrients to the leaves for the maintenance of photosynthesis being used by larvae (Whitham, 1979). Only two species presented galls in stems, Andira cujabensis and $A$. fraxinifolia. In both cases, the inducer is unidentified at species level.

The Southeast region is the best sampled in Brazil, because in this region were made the first studies on ecological and taxonomic aspects of gall-inducing insects and also where the first training centers of researchers were developed. New sampling efforts for gall-inducing insects need to be made throughout Brazil, because even in Southeast, the majority of gall-inducing species are unknown, despite the efforts made in the last 30 years (Araújo et al., 2019).

The high resolution photos of the exsiccates provided by the virtual herbaria and in the inventories made possible to detect new records of occurrence for six morphotypes of galls and gall inducers in four Andira species: three new registers for the fusiform gall in $A$. fraxinifolia, four registers for the fusiform gall in A. humilis, two new registers for the lenticular gall in $A$. nitida, and six new registers for three morphotypes in $A$. vermifuga. No new records were found for the morphotypes of $A$. cujabensis and $A$. surinamensis.

A. fraxinifolia has distribution registered in seventeen Brazilian states, in eight of the nine states of the Northeast region, two in the Midwest region (GO and MS), and in all states of the Southeast and South regions (Ramos et al., 2020) (Fig. 2). On the other hand, the morphotypes that occur in this species were found in only seven Brazilian states, two in the Northeast (one new in Bahia), all states of the Southeast region, one in South region (Santa Catarina, new record) and no one in the Midwest region (Fig. 2).
A. humilis has distribution registered in eight Brazilian states (Fig. 4), but the morphotype of this species were found in five of them, two in the Northeast (one new to Paraíba, where the species was not recorded either), two in the Southeast (new to Minas Gerais with three new points of occurrence), and one in South region (Fig. 4).

A. nitida has a restricted distribution to six states of the Northeast region and Espírito Santo, in the Southeast region, all located on the East Coast of Brazil (Fig. 6); with the lenticular morphotype found in two Brazilian states, one in the Northeast (Bahia, new register) and the other in the Southeast (Espírito Santo, new point of occurrence) (Fig. 6).

$A$. vermifuga has been registered or has records in eleven Brazilian states in all regions, except for the South (Fig. 9). Three morphotypes have new registers: the globoid induced by Lopesia chapadensis (one in Ceará State, two in Goiás and one in the Federal District), and the lenticulars 2 and 3 with new points of occurrence in Mato Grosso and Goiás, respectively (Fig. 9).

Although the six Andira species studied are distributed in large regions of Brazil, we did not find records of gall morphotypes for all Brazilian states in which the species are registered, indicating that the distribution of gall inducers depends on other factors besides the presence of the host plant. Anyway, after our searches, the points of occurrence of gall morphotypes in Andira species passed from 15 to 30, the occurrence in states, from 12 to 17, plus the Federal District (Fig. 11).

Some actions could be adopted by herbaria to increase the possibility of detecting gall morphotypes. Not all exsiccates present in the herbaria are available in the online database, limiting the results. A low number of exsiccates with galls or herbivore marks are deposited, possibly due to the storage criteria of the herbaria, which tend to select samples without insect damages, that could potentially contaminate other herbarium samples. Even though, these digital tools help to increase the records of cecidomyiids through the recognition of their morphotypes, because the morphotypes found in exsiccates are reliable markers of the gall inducers' presence in locations are still unpublished. It is possible to perceive the great potential of the use of online herbaria for studies of expansion of the geographical distribution of plant species, and also to the species-specific interaction between host plants and gall inducers.

Even considering that the storage and conservation of exsiccates have a bias that often avoids gall records in herbariums and that there are many regions of Brazil little explored by inventories with the main objective of finding galls and their inducers, our results indicate the distribution of gall inducers depends on other factors besides the presence of the host plant that need to be investigated in new studies.

\section{Acknowledgements}

The authors acknowledge the curators of the herbaria who provide exsiccates online: Taciana Barbosa Cavalcanti e Bruno Machado Teles Walter (CEN), Rafaela Campostrini Forzza (JBJR), David Laughlin(K), Helio de Queiroz Boudet Fernandes (MBML), Vanessa Invernon(MNHN), Matthew Pace (NY), Lívia Cordi (UEC), and Antônio Laffayete Pires da Silveira (RON).

\section{Conflicts of interest}

The authors declare no conflicts of interest.

\section{Author contribution statement}

MVUG - Substantial contribution in the concept and design of the study, data analysis and interpretation, manuscript preparation, critical revision, adding intellectual content; EAMS - Substantial contribution in the data collection, data analysis and interpretation, manuscript preparation, critical revision, adding intellectual content. 


\section{References}

Almada, E.D., Wilson, G., Fernandes, A., 2011. Insetos indutores de galhas em florestas de terra firme e em reflorestamentos com espécies nativas na Amazônia Oriental, Pará, Brasil. Bol. Mus. Para. Emílio Goeldi Ciênc. Nat. 6, 163-196.

Araújo, W.S., Sobral, F.L., Maracahipes, L., 2014. Insect galls of the Parque Nacional das Emas (Mineiros, GO, Brazil). Check List 10, 1445-1451. https://doi.org/10.15560/10.6.1445.

Araújo, W. S., Fernandes, G. W., Santos, J. C., 2019. An overview of inventories of gall-inducing insects in Brazil: looking for patterns and identifying knowledge gaps. An. Acad. Bras. Cienc. 91 (1), e20180162. https://doi.org/10.1590/0001-3765201920180162 (accessed 20 December 2020).

Araújo, W.S., Urso-Guimarães, M.V., Mendonça, M.S., Santos, J.C., 2021. Sampling Galls and Galling Arthropods. In: Santos, J.C., Fernandes, G.W. (Eds.), Measuring Arthropod Biodiversity. Springer, Cham, pp. 403-437.

Arriola, I.A., de Melo Júnior, J.C.F., Mouga, D.M.D.S., Isaias, R.M. S., Costa, E.C., 2016. Where host plant goes, galls go too: new records of the Neotropical galling Cecidomyiidae (Diptera) associated with Calophyllum brasiliense Cambess. (Calophyllaceae). Check List 12 (4), 1-8. https://doi.org/10.15560/12.4.1924.

Arriola, Í.A., de Melo Júnior, J.C.F., 2016. Richness of insect galls on shrub-tree restinga of a coastal plain of southern Brazil. Acta Biol. Catarinense. 5 (1), 56-64. https://doi.org/10.21726/abc.v3i2.288.

Bergamini, B.A.R., Bergamini, L.L., Santos, B.B., Araújo, W.S., 2017. Occurrence and characterization of insect galls in the Floresta Nacional de Silvânia, Brazil. Pap. Avulsos Zool. 57 (32), 413-431. https://doi.org/10.11606/0031-1049.2017.57.32.

Bregonci, J.M., Polycarpo, P.V., Maia, V.C., 2010. Galhas de insetos do Parque Estadual Paulo César Vinha (Guarapari, ES, Brasil). Biota Neotrop. 10, 265-274.

Carneiro, M.A.A., Branco, C.S.A., Braga, C.E.D., Almada, E.D., Costa, M.B.M., Maia, V.C., Fernandes, G.W., 2009. Are gall midge species (Diptera, Cecidomyiidae) host-plant specialists? Rev. Bras. Entomol. 53, 365-378. https://doi.org/10.1590/s0085-56262009000300010.

Carvalho-Fernandes, S.P., Ascendino, S., Maia, V.C., Couri, M.S., 2016. Diversity of insect galls associated with coastal shrub vegetation in Rio de Janeiro, Brazil. An. Acad. Bras. Cienc. 88, 1407-1418. https:// doi.org/10.1590/0001-3765201620150658.

Cuevas-Reyes, P., Quesada, M., Hanson, P., Dirzo, R., Oyama, K., 2004. Diversity of gall-inducing insects in a Mexican tropical dry forest: the importance of plant species richness, life-forms, host plant age and plant density.J. Ecol. 92, 707-716. https://doi.org/10.1111/j.00220477.2004.00896.x.

Espírito-Santo, M.M., Fernandes, G.W., 2007. How many species of gall-inducing insects are there on earth, and where are they? Ann. Entomol. Soc. Am. 100, 95-99. https://doi.org/10.1603/00138746(2007)100[95:hmsogi]2.0.co;2.

Gagné, R.J., Jaschhof, M., 2021. A Catalog of the Cecidomyiidae (Diptera) of the World. 5th ed. 813 pp. Available in: https://www.ars.usda.gov/ ARSUserFiles/80420580/Gagne_Jaschhof_2021_World_Cat_5th_Ed.pdf (accessed 18 June 2021).

Garcia, C.A., Urso-Guimarães, M.V., 2018. Three New Species of Lopesia Rübsaamen (Diptera: Cecidomyiidae) from Brazil. Fla. Entomol. 101, 203-211. https://doi.org/10.1653/024.101.0208.

Garcia, C.A., Lima, V.P., Calado, D.C., Urso-Guimarães, M.V., 2017. New species of Lopesia Rübsaamen (Diptera: Cecidomyiidae) associated with Andira humilis Mart. ex Benth. (Fabaceae). Rev. Bras. Entomol. 61, 239-242. https://doi.org/10.1016/j.rbe.2017.06.001.
IBGE, 2019. Bases cartográficas contínuas. Available in: https://www.ibge. gov.br/geociencias/cartas-e-mapas/bases-cartograficas-continuas/ (accessed 6 November 2019).

Isaias, R.M.S., Oliveira, D.C., Carneiro, R.G.S., Kraus, J.E., 2014. Developmental anatomy of galls in the Neotropics: arthropods stimuli versus host plants constraints. In: Fernandes, G.W., Santos, J.C. (Eds.), Neotropical Insect Galls. Springer Verlag, New York, pp. 51-67.

Lima, V.P., Calado, D., 2018. Morphological characterization of insect galls and new records of associated invertebrates in a Cerrado area in Bahia state, Brazil. Braz. J. Biol. 78, 636-643. https://doi. org/10.1590/1519-6984.169502.

Lima, V.P., Calado, D.C., 2020. Mapping the habitat suitability of Andira humilis Mart. ex Benth. (Fabaceae) as a means to detect its associated galling species in Brazil. Acta Sci. Biol. Sci. 42, 1-7. https://doi. org/10.4025/actascibiolsci.v42i1.48809.

Luz, G.R., Fernandes, G.W., Silva, J.O., Neves, F.S., Fagundes, M., 2012. Galhas de insetos em habitats xérico e mésico em região de transição Cerrado-Caatinga no norte de Minas Gerais, Brasil. Neotrop. Biol. Conserv. 7, 171-187. https://doi.org/10.4013/nbc.2012.73.04.

Maia, V.C., 2021. Cecidomyiidae (Diptera, Insecta): richness of species and distribution in Brazil. Biota Neotrop. 21 (2), e20201038.

Maia, V.C., Azevedo, M.A.P., Couri, M.S., 2002. New contribution to the knowledge of the gall midges (Diptera, Cecidomyiidae) from the restinga of Barra de Maricá(Rio de Janeiro, Brazil). Stud. Dipterol. 9 (2), 447-452.

Maia, V.C., Cardoso, L.J.T., Braga, J.M.A., 2014. Insect galls from Atlantic Forest areas of Santa Teresa, Espírito Santo, Brazil: characterization and occurrence. Bol. Mus. Biol. Mello Leitao. 33, 47-129.

Maia, V. C., Fernandes, G. W., 2004. Insect galls from Serra de São José (Tiradentes, MG, Brazil). Braz. J. Biol. 64, 423-445.

Maia, V.C., Magenta, M.A.G., Martins, S.E., 2008. Ocorrência e caracterização de galhas de insetos em áreas de restinga de Bertioga (São Paulo, Brasil). Biota Neotrop. 8, 167-197. https://doi.org/10.1590/S167606032008000100020.

Maia, V.C., de Oliveira, L.A., 2018. Lopesia indaiensis (Diptera, Cecidomyiidae), a new species of gall midge feeding on Andira fraxinifolia Benth (Fabaceae), an endemic plant in Brazil. Rev. Bras. Entomol. 62, 125-130. https://doi.org/10.1016/j.rbe.2018.01.001.

Mani, M.S. (1964). Ecology of Plant Galls. Springer-Science+Business Media, B.V., USA.

Pennington, R.T., 2003. Monograph of Andira (Leguminosae-Papilionoideae). Syst. Bot. Monogr. 64, 1-143. https://doi.org/10.2307/25027903.

QGIS Development Team, 2020. QGIS Geographic Information System. Open Source Geospatial Foundation Project. Available in: http:// qgis.osgeo.org (Accessed 13 October 2020).

Ramos, G., Cardoso, D.B.O.S., Pennington, R.T., 2020. Andira in Flora do Brasil 2020. Jardim Botânico do Rio de Janeiro. Available in: http:// floradobrasil.jbrj.gov.br/reflora/floradobrasil/FB29442 (accessed 12 March 2021).

Rodrigues, A.R., Maia, V.C., Couri, M.S., 2014. Insect galls of restinga areas of Ilha da Marambaia, Rio de Janeiro, Brazil. Rev. Bras. Entomol. 58, 173-197. https://doi.org/10.1590/S0085-56262014000200010.

Saito, V.S., Urso-Guimarães, M.V., 2012. Characterization of galls, insect galls and associated fauna of Ecological Station of Jataí (Luiz Antônio, SP). Biota Neotrop. 12, 99-107. https://doi.org/10.1590/ s1676-06032012000300011.

Santos, B.B., Ribeiro, B.A., Silva, T.M., Araújo, W.S., 2012a. Galhas de insetos em uma área de cerrado sentido restrito na região semi-urbana de Caldas Novas (Goiás, Brasil). Rev. Bras. Biociencias. 10, 439-445.

Santos, J.C., de Almeida-Cortez,J.S., Fernandes, G.W., 2012b. Gall-inducing insects from Atlantic Forest of Pernambuco, Northeastern Brazil. Biota Neotrop. 12, 196-212. https://doi.org/10.1590/s1676-06032012000300020. 
Santos-Silva, J., Araújo, T.J., 2020. Are Fabaceae the principal superhosts of galls in Brazil? An. Acad. Bras. Cienc. 92, 1-15. https://doi. org/10.1590/0001-3765202020181115.

Shorthouse, J.D., Rohfritsch, O., 1992. Biology of Insect-Induced Galls. Oxford University Press, New York.

Shorthouse, J.D., Wool, D., Raman, A., 2005. Gall-inducing insects Nature's most sophisticated herbivores. Basic Appl. Ecol. 6, 407-411. https://doi.org/10.1016/j.baae.2005.07.001.

Skuhravá, M., Skuhravý, V., 2009. Species richness of gall midges (Diptera: Cecidomyiidae) in Europe (West Palaearctic): biogeography and coevolution with host plants. Acta Soc. Zool. Bohem. 73, 87-156.

Skuhravá, M., Skuhravý, V., Meyer, H., 2014. Gall midges (Díptera: Cecidomyiidae: Cecidomyiinae) of Germany - Faunistics, ecology and zoogeography. Faun.-Ökol. Mitt. (Suppl. 38), 1-200.
Stone, G.N., Schönrogge, K., 2003. The adaptive significance of insect gall morphology. Trends Ecol. Evol. 18, 512-522. https://doi.org/10.1016/ S0169-5347(03)00247-7.

Tavares, J. S., 1920. Cecidologia brazileira: cecídias que se criam em plantas das famílias das Leguminosae, Sapotaceae, Lauraceae, Myrtaceae, Punicaceae, Aurantiaceae, Malpighiaceae, Sapindaceae, Umbelliferae, Loranthaceae, Apocynaceae, Urticaceae, Salicaceae e Gramineae. Brotéria: Serie Zoologica 18 (2), 82-96.

Veldtman, R., McGeoch, M.A., 2003. Gall-forming insect species richness along a non-scleromorphic vegetation rainfall gradient in South Africa: the importance of plant community composition. Austral Ecol. 28, 1-13. https://doi.org/10.1046/j.1442-9993.2003.01234.x. Whitham, T.G., 1979. Territorial behavior of Pemphigus gall aphids. Nature. 279, 324-325. https://doi.org/10.1038/279324a0. 